\title{
Pattern selection in reaction diffusion systems
}

\author{
Srikanth Subramanian @ and Seán M. Murray* \\ Max Planck Institute for Terrestrial Microbiology, 35043 Marburg, Germany
}

(Received 28 August 2020; accepted 7 January 2021; published 25 January 2021)

\begin{abstract}
Turing's theory of pattern formation has been used to describe the formation of self-organized periodic patterns in many biological, chemical, and physical systems. However, the use of such models is hindered by our inability to predict, in general, which pattern is obtained from a given set of model parameters. While much is known near the onset of the spatial instability, the mechanisms underlying pattern selection and dynamics away from onset are much less understood. Here, we provide physical insight into the dynamics of these systems. We find that peaks in a Turing pattern behave as point sinks, the dynamics of which is determined by the diffusive fluxes into them. As a result, peaks move toward a periodic steady-state configuration that minimizes the mass of the diffusive species. We also show that the preferred number of peaks at the final steady state is such that this mass is minimized. Our work presents mass minimization as a potential general principle for understanding pattern formation in reaction diffusion systems far from onset.
\end{abstract}

DOI: 10.1103/PhysRevE.103.012215

\section{INTRODUCTION}

Pattern formation occurs in a huge variety of natural and living systems [1], from chemical reactions [2,3] to living cells [4-6] to environmental patterns [7]. In systems described by reaction diffusion (RD) equations, the formation of spatially periodic patterns can be explained by the Turing instability, in which patterns emerge due to the presence of two or more interacting components that diffuse (or are transported) at different rates [8-11]. The resulting patterns are multistable in that several different stable patterns can be obtained from the same set of parameters, albeit, for incompletely understood reasons, with different frequencies [12].

Sufficient conditions for pattern formation can be determined in the so-called Turing or linear regime, in which a spatially uniform stable steady state becomes linearly unstable to spatial perturbations in the presence of diffusion [9]. Consider the following one-dimensional system:

$$
\begin{aligned}
& \partial_{t} u=D_{u} \partial_{x}^{2} u+f(u, v), \\
& \partial_{t} v=D_{v} \partial_{x}^{2} v+g(u, v) .
\end{aligned}
$$

The evolution of any small perturbation from a spatially uniform steady state is given by its decomposition into its Fourier modes $e^{\sigma_{k} t} \cos (k x)$, where $\operatorname{Re}\left(\sigma_{k}\right)$ is the growth rate. Then, the uniform steady state is laterally unstable if any wave number $k$ has a positive growth rate $\operatorname{Re}\left(\sigma_{k}\right)>0$ [see Fig. 1(a) and the Supplemental Material [13]). For a finite domain $[0, L]$

*sean.murray@ synmikro.mpi-marburg.mpg.de

Published by the American Physical Society under the terms of the Creative Commons Attribution 4.0 International license. Further distribution of this work must maintain attribution to the author(s) and the published article's title, journal citation, and DOI. Open access publication funded by the Max Planck Society. and reflective boundary conditions, the wave number $k$ is discrete with $k=\frac{n \pi}{L}$ for integer $n$. The unstable modes grow exponentially in time until the nonlinear terms can no longer be neglected. These terms saturate the exponential growth and select different spatial states. At the onset of the instability when a single mode $n_{c}$ is unstable, the naive expectation is that the growth saturates without substantially changing the spatial structure so that the final state is qualitatively similar to mode $n_{c}$. This saturation is described by the corresponding amplitude equations $[10,14,15]$. These equations and, more generally, the weakly nonlinear approach on which they are based, have been extremely useful in understanding the selection and stability of different fundamental modes in a variety of systems [10,14-18], as well as the effect of external constraints such as fixed boundary conditions, parameter ramps, external forcing, template patterns, system geometry, and deformable or moving boundaries [19-29].

However, the approach is only valid close to onset (in the vicinity of the bifurcation). Away from onset, where many modes are linearly unstable [Fig. 1(a)], pattern-forming RD systems typically still produce patterns with a well-defined periodicity, corresponding to [in one dimension (1D)] a particular mode number $n$ and its harmonics $2 n, 3 n, \ldots$ [Fig. 1(b)]. This is despite neighboring modes $n \pm 1$ generally having similar growth rates, which would be expected to lead to aperiodic patterns. The physics underlying this "exclusion principle" [19] are in general not known. ${ }^{1}$ This is very relevant as nonequilibrium systems in nature cannot be expected to be close to onset. For the special case (see below) of twocomponent mass-conserved models, Lyanpunov functionals have been used to demonstrate that the only stable nontrivial solution consists of a single peak or half-peak [30-33].

${ }^{1}$ See Sec. 9.1.4 of [10] for a more detailed discussion. 

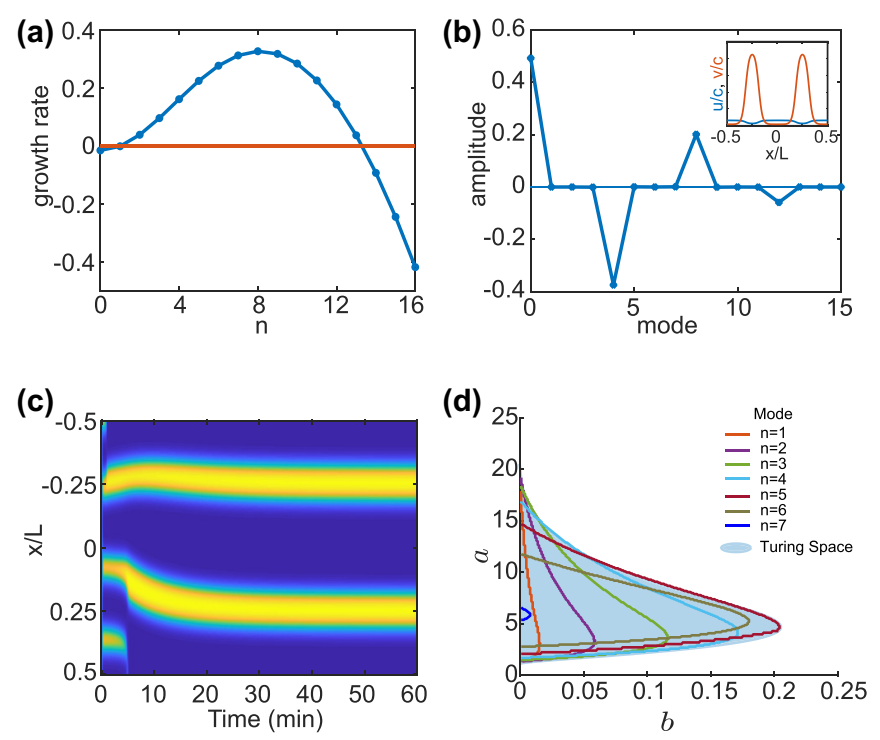

FIG. 1. The Turing instability of reaction diffusion systems. (a) The growth rate of different modes for the model in (2) with default parameters and $L=4$. Note that the growth rate at $n=0$ is negative: the system is not generically mass conserving. (b) The Fourier decomposition of the obtained two-peak pattern (inset). (c) An example kymograph showing pattern development starting from a random perturbation of the uniform state. While mode $n=7$ dominates initially, the pattern coarsens down to two peaks, dominated by mode $n=4$. See also Fig. S1 [13]. (d) The regions of instability of each mode on a domain of length $L=2$ (the region bounded by the $y$ axis and the corresponding colored curve). The blue shaded region shows the Turing space for an infinite domain (see also Fig. S1 and the Supplemental Material [13] for further details).

Unfortunately, the construction of such functionals is challenging for reaction diffusion systems in general.

In the following, we propose a simple physical principle to explain the dynamics, positioning, and number of peaks in a Turing pattern far from onset. Inspired by our previous work [12], we begin by reviewing a number of observations about patterns on a 1D domain. As already mentioned, final patterns away from onset still have a well-defined wavelength. With reflective boundary conditions, the peaks are also regularly positioned, i.e., the peaks (or valleys) of the pattern are found at the same locations as those of some fundamental mode $n$. While this regular positioning is consistent with the selection of a particular mode, it appears, for the reasons given above, that this is a nonlinear effect.

We will also see below that if a system is initialized with a mispositioned pattern (and therefore far outside of the linear regime), for example, a single mispositioned peak, then the peak subsequently moves towards mid-domain without substantially changing its shape. This is also evident in models that exhibit coarsening [12,34-37], which we define here as the preference for a steady-state pattern dominated by a mode lower than that predicted by linear stability, i.e., the mode with greatest linear growth rate $\sigma$. In Fig. 1(c), we show the evolution of a pattern starting from a small perturbation of the uniform state. The pattern initially resembles mode $n=7$ (three and a half peaks) consistent with the linear prediction
[Fig. 1(a)] but it subsequently coarsens, first to three peaks, and then to two. After each coarsening event, the peaks move towards their regularly positioned configuration so that the final steady-state pattern consists of a peak at each quarter position (mode 4). In two-variable mass-conserved systems, coarsening is complete in that, irrespective of how many peaks there are initially, the pattern eventually coarsens down to a single peak or half-peak (monotonic) solution [30-33,38]. On a periodic domain, absolute positioning is no longer meaningful but peaks still reposition to maintain a constant wavelength. Finally, regular positioning is maintained even during domain growth in which new peaks are created by insertion or splitting [11,12,39]. Overall, these observations indicate that the periodic positioning of peaks is an inherently nonlinear effect and not a remnant nor direct consequence of the dominating linear mode of the base state perturbation. Thus, while a decomposition into fundamental modes is critical to understanding the initial formation of the pattern (starting from the homogeneous state), once peaks have formed, a different description is required.

Note also that the two phases of a pattern that exist when imposing reflective boundary conditions are not necessarily equally preferred. We have previously studied a model in which the pattern consisting of a single peak at mid-domain is preferred over a half-peak at each boundary and similarly for higher modes [12]. Thus, not only is the mode of the pattern selected, the phase is too. However, peaks on the boundary display different dynamics: unlike interior peaks, they do not move but only appear or disappear. Here, we will restrict ourselves to the study of interior peaks only as they are amenable to comparison with point sinks and hence we will not address the issue of phase selection. We make this explicit in the last section by using periodic boundary conditions. In the interim, we will use reflective boundary conditions in order to more easily study peak movement.

In the following, we show that the peaks of a Turing pattern behave as point sinks that move with a velocity proportional to the diffusive flux across them. This is a consequence of the flow of mass through the system and is responsible for the regular positioning of peaks. By flow, we mean something more than simply the flux through the system. In a diffusive non-mass-conserving system, "molecules" enter the system, diffuse, and either leave the system or are converted to another species. This combination of diffusion and turnover results, as we shall see, in the regular positioning of peaks due to the concept of flux balance [40,41]. This result also explains why the peaks in mass-conserving two-variable reaction diffusion systems do not move: there is no flow to drive the movement. In our analysis, we use singular perturbation methods to study solutions consisting of well-separated spikes, a regime that occurs in the singular limit $D_{v} \rightarrow 0$ of reaction diffusion systems (see [42] for an overview). Similar approaches have been used to study traveling pulse and kink solutions in other pattern-forming systems [43-48] and, more recently, to study peak splitting [49].

We also find that the regularly positioned configuration minimizes the total mass of the rapidly diffusing species, the substrate of the nonlinear reaction. We then find empirically that this "minimization principle" can be extended to predict not only the final positions of the peaks, but also the final 
number of peaks, even in the presence of coarsening. This is significant as the amplitude equation approach for determining the dominant mode is not applicable far from onset. Mass minimization therefore has the potential to be an incredibly simple yet powerful concept for understanding the behavior of pattern-forming reaction diffusion systems.

\section{MODEL}

We introduce the following exploratory one-dimensional system, inspired by our recent model of bacterial condensin $[12,50]$, written in terms of the variables $u=u(x, t)$ and $v=$ $v(x, t)$,

$$
\begin{aligned}
& \partial_{t} u=D_{u} \partial_{x}^{2} u-\beta u(u+v)^{2}+\gamma v+c \delta-\delta u, \\
& \partial_{t} v=D_{v} \partial_{x}^{2} v+\beta u(u+v)^{2}-\gamma v-\delta v,
\end{aligned}
$$

defined over the spatial domain $[-L / 2, L / 2]$, with reflective boundary conditions, all parameters non-negative and $D_{v}<$ $D_{u}$. While superficially similar to the some of the classic Turing models such as the Brusselator [51] and Schnakenberg [52] models, this model has some notable properties. In the absence of diffusion, it has a single fixed point that is stable for all parameter values. This means that the stability diagram of the system is particularly simple. There are only two regions, specified by a single inequality: one in which the spatially uniform solution is stable and another in which it is Turing unstable [Fig. 1(d)]. There are no oscillatory instabilities. Like the Brusselator, the model has the form of a mass-conserving Turing system with additional terms: a global source term $c \delta$ and two depletion terms $\delta u$ and $\delta v$. By writing the source term as $c \delta$, we can change $\delta$, the turnover rate, while leaving the total steady-state concentration $c$ fixed. We obtain a massconserved Turing model when $\delta=0$ and the limit $\delta \rightarrow 0$ is well defined as long as we constrain the total initial mass to be the same as the steady-state mass, i.e., $C(0)=c$.

The condition for a Turing instability is most easily stated by nondimensionalizing the system and introducing the dimensionless parameters $a=\frac{\beta c^{2}}{\gamma}, b=\frac{\delta}{\gamma}, \Gamma=\frac{\gamma L^{2}}{D_{v}}, d=\frac{D_{u}}{D_{v}}$ (see Supplemental Material [13] for details). As can be seen in Fig. 1(d) for typically choices of the diffusivity ratio $d$, we require $b \ll 1$ for patterning, i.e., the timescale of mass flow (turnover) through the system $1 / \delta$ must be much longer than the timescale underlying the Turing instability $1 / \gamma$.

Numerically solving the system, we found that it indeed produces regularly positioned peaks. We also observed that, like the model it is based on [12], it exhibits a competition instability [35-37] (also known as interrupted coarsening [34]) in that the final dominant mode has a longer wavelength than predicted by linear stability analysis. For our default parameter set with $L=4(\Gamma=4800)$, linear stability predicts [Fig. 1(a)] that the pattern consists of four peaks (or valleys) (mode $n=8$ ) whereas the obtained steady-state pattern most frequently consists of two peaks (mode $n=4$ ) [Figs. 1(b) and S1D [13]]. While multiple peaks often form initially, consistent with the linear prediction, coarsening rapidly occurs, leaving mispositioned peaks that then move slowly towards opposite quarter positions, while maintaining their shape [Fig. 1(c)]. Note that this movement is only observed because of the competition instability. It is not evident in models or (a)
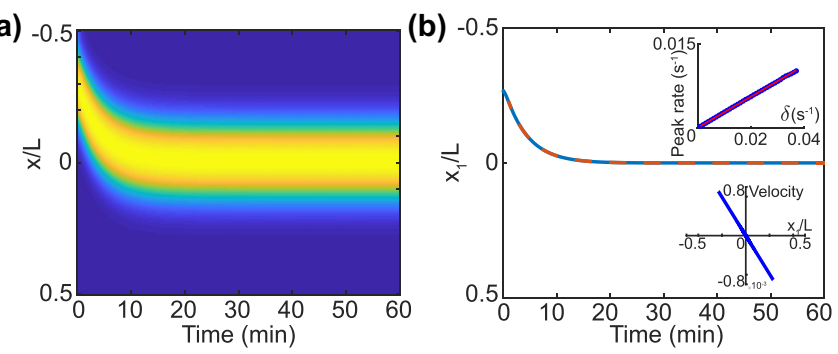

(c)

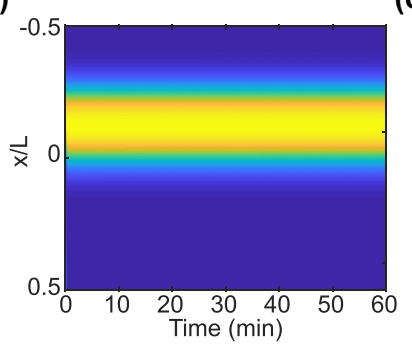

(d)

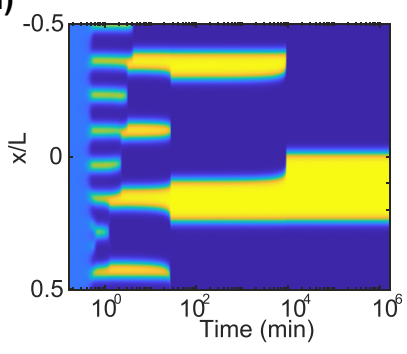

FIG. 2. Peak movement and regular positioning depend on flux through the system. (a) The system is initialized with a peak away from mid-domain. The peak subsequently moves to mid-domain. (b) The centroid of the peak (blue line) plotted as function of simulation time. The orange dashed line is an exponential fit. Inset: (Top right) The rate of movement obtained from fitting the centroid to an exponential as in (b) shows a linear dependence on the turnover rate $\delta$. (Bottom right) Peak velocity is linear in peak position. (c) A single peak in the mass-conserved limit $\delta=0$ can be positioned anywhere on the domain. No peak movement is observed. (d) The mass-conserved system exhibits complete coarsening. Irrespective of how many peaks there are initially, the pattern eventually coarsens to a single peak, the position of which depends on which peak of the initial pattern has not coarsened. In (d) $\Gamma=19200(L=10)$.

parameters sets for which the linear prediction holds as in that case, the peaks are created at their steady-state positions. We will return to this incomplete coarsening later.

To examine the movement of peaks in more detail, we focused on the case of a single peak $(n=2)$, typically obtained for $L=2(\Gamma=1200)$. Examining the movement of the peak [Fig. 2(a)], we found that it moves to mid-domain exponentially in time [Fig. 2(b)], indicating the peak velocity is linearly proportional to its displacement from mid-domain [Fig. 2(b), bottom inset]. This was the case whether the system was initialized with a random perturbation of the uniform state or with a peak preformed somewhere on the domain. That peaks might move to respect the symmetry of the system is perhaps, while underappreciated, not surprising though it is relevant for understanding the periodicity and positioning of peaks. Indeed, the rate of movement was found to be directly proportional to the turnover rate $\delta$ [Fig. 2(b), top inset] (or equivalently $c \delta$ the flux through the system per unit length) so that in the mass-conserved limit $\delta \rightarrow 0$, peaks do not move [Figs. 2(b) and 2(c)]. This is consistent with our previous results [12]. Mass-conserving RD models exhibit a complete coarsening process in that the final steady-state pattern is either monomodal (periodic or reflective) or monotonic (reflective only) depending on the boundary conditions, as has been proved explicitly for several models [30-33,38]. We find 
the same coarsening behavior here [Fig. 2(d)]. We only obtain the half-peak solution for very short domains, i.e., when the width of the interface is comparable to the domain length. If the domain length or other parameters are chosen such that there is initially more than one peak, then the coarsening process results (eventually) in a single interior peak [Fig. 2(d)]. Importantly, since peaks do not move, the position of this final peak is determined by whichever peak of the transient state remains after coarsening, i.e., the steady-state solutions are in general not symmetric as might naively be expected by the boundary conditions. We tested these conclusions by initializing the system with a single preformed peak (constructed as a translation of the non-mass-conserved steady-state solution). We found that preformed peaks do not move and constitute a stable solution [Fig. 2(c)]. Thus, the mass-conserved case $b=0$ with reflective boundary conditions has a continuum of single-peak stable states, whereas there is at most one unique single-peak solution for $b>0$. This implies that regular positioning is not an intrinsic property of the system but rather depends on $b$. These results are based on simulations that were run for very long times with very low error tolerances and are in agreement with our previous results [12]. We will also see the same behavior when we consider point sinks in the next section. Overall, these results demonstrate a connection between peak movement towards the regular positioned configuration and the flow (turnover) of mass through the system.

\section{POINT SINKS}

To explore this connection in more detail, we turn to a toy model involving diffusion and point sinks. We consider the steady-state diffusion equation for a variable $A=A(x)$ over a one-dimensional domain of length $L$ in the presence of global source and decay terms as well as $n$ localized point sinks at positions $\boldsymbol{x}=\left(x_{1}, \ldots, x_{n}\right)$ (each with rate $\left.\mu\right)$ :

$$
D \frac{d^{2} A}{d x^{2}}+c \delta-\delta A-\sum_{i=1}^{n} \mu L \delta\left(x-x_{i}\right) A=0 .
$$

We take the domain to be $[-L / 2, L / 2]$ and impose zero-flux boundary conditions. As before, we write the global source term in terms of the decay rate $\delta$ and a concentration $c$, which is the steady-state concentration in the absence of the point sinks. A simpler system without the decay term and with perfect points sinks (i.e., $\mu \rightarrow \infty$ ) was used by Ietswaart et al. to model the positioning of plasmids within rod-shaped bacterial cells [41]. They found that the gradient differential across each sink vanishes if and only if the sinks are regularly positioned and, therefore, if sinks were to move up the concentration gradient, they would be regularly positioned. We will extend this result to the more complicated case of (3). Note that the presence of the decay term introduces an additional length scale $\sqrt{D / \delta}$ into the system, namely, the distance that a molecule of $A$ would diffuse (in the absence of any point sinks) before it decays. We refer to this as the length scale of diffusion. It is small when either diffusion is slow or the decay rate (turnover) $\delta$ is fast.

We can write the solution to (3) as

$$
A(x)=c-\sum_{i} \mu_{i}^{\prime} G\left(x ; x_{i}\right)
$$

where $G\left(x ; x_{i}\right)$ is the modified Green's function defined by

$$
\begin{aligned}
-\frac{L^{2}}{\kappa^{2}} G_{x x}\left(x ; x_{i}\right)+G\left(x ; x_{i}\right) & =L \delta\left(x-x_{i}\right) \\
G_{x}\left( \pm \frac{L}{2} ; x_{i}\right) & =0, \quad \frac{1}{L} \int_{-\frac{L}{2}}^{\frac{L}{2}} G\left(x ; x_{i}\right) d x=1
\end{aligned}
$$

where the dimensionless parameter $\kappa=L \sqrt{\frac{\delta}{D}}$ is the ratio of the length of domain to the length scale of diffusion. The coefficients $\mu_{i}^{\prime}=\mu_{i}^{\prime}(\boldsymbol{x})$ are determined by the linear algebraic conditions

$$
\mu_{i}^{\prime}=\lambda A\left(x_{i}\right), \quad i=1, \ldots, n
$$

where we have defined a second dimensionless parameter $\lambda=\frac{\mu}{\delta}$, the ratio of the sink and background decay rates. The quantities $\mu_{i}^{\prime}$ have a simple interpretation. They are directly related to $J_{i}$, the flux leaving the system through each sink:

$J_{i}=J_{i+}+J_{i-}=-D \sum_{j} \mu_{j}^{\prime}\left[G_{x}\left(x_{i}^{+} ; x_{j}\right)-G_{x}\left(x_{i}^{-} ; x_{j}\right)\right]=L \delta \mu_{i}^{\prime}$,

where $J_{i}=\left|D \frac{d A}{d x}\right|$ and the - and + subscripts refer to the diffusive flux from the left and right, respectively. We also define the flux differential across each sink as

$$
\begin{aligned}
\Delta J_{i} & =\frac{1}{2}\left(J_{i+}-J_{i-}\right) \\
& =-\frac{D}{2} \sum_{j} \mu_{j}^{\prime}\left[G_{x}\left(x_{i}^{+} ; x_{j}\right)+G_{x}\left(x_{i}^{-} ; x_{j}\right)\right] .
\end{aligned}
$$

Note the total mass (concentration) of $A$ in the system is readily given by

$$
M:=\frac{1}{L} \int_{-\frac{L}{2}}^{\frac{L}{2}} A(x) d x=c-\sum_{i} \mu_{i}^{\prime},
$$

where the second term solely describes the effect of the point sinks.

We can now investigate what would in happen in this system if sinks were to move up the gradient of $A$. As in Ietwaart et al., we can determine the configurations for which the flux differentials are all zero. In Appendix A, we prove that this occurs for regularly positioned sinks $x_{i}=\bar{x}_{i}:=\left(i-\frac{1}{2}\right) \frac{L}{n}-\frac{L}{2}$, i.e.,

$$
\Delta J_{i}(\overline{\boldsymbol{x}})=0 \quad \text { for all } i
$$

and, interestingly, we also show that the regular positioned configuration $\overline{\boldsymbol{x}}$ is a stationary point of the mass $M$, i.e.,

$$
\left.\frac{\partial}{\partial x_{i}} M(\boldsymbol{x})\right|_{\boldsymbol{x}=\overline{\boldsymbol{x}}}=0 \text { for all } i
$$

Based on our numerical observations, we conclude that this stationary point is generically a minimum (see Appendix A).

Thus, if sinks move up the concentration gradient (in the direction of greatest flux), they will be regularly positioned as this is the configuration for which the fluxes into each sink from either side balance. Furthermore, this configuration minimizes the total mass of the system. In other words, the sinks are positioned so as to "consume" mass at the greatest rate. This connection between regular positioning and mass 
minimization appears to be generalizable and we have observed numerically that it holds for spatial sinks, i.e., if the delta function in (3) is replaced by a peak-shaped spatial function such as a Gaussian function or $\operatorname{sech}^{2}(x)$, then the total mass is minimized when the sink is centered at mid-domain.

Let us consider the case of a single sink, $n=1$, in more detail. We focus on the regime $\kappa \ll 1$ in which the diffusive length scale is much longer than the domain size. We expand in $\kappa$ to find first

$$
\frac{\mu_{1}^{\prime}}{c} \approx \frac{\lambda}{\lambda+1}-\frac{\lambda^{2}}{\lambda+1}\left(\frac{x_{1}^{2}}{L^{2}}+\frac{1}{12}\right) \kappa^{2}+O\left(\kappa^{4}\right)
$$

and then

$$
\begin{aligned}
\frac{\Delta J_{1}}{c \delta L} & =-\frac{1}{2} \frac{\mu_{1}^{\prime}}{c} \frac{\sinh \left(2 \kappa \frac{x_{1}}{L}\right)}{\sinh (\kappa)} \\
& \approx-\frac{\lambda}{\lambda+1} \frac{x_{1}}{L}+O\left(\kappa^{2}\right) .
\end{aligned}
$$

Hence, if $\kappa \ll 1$, then the flux differential across the sink depends linearly on its relative displacement from mid-domain. For strong sinks $(\lambda \gg 1)$, the proportionality factor is linear in $\delta$, just as we observed for the Turing system [Fig. 2(b)]. As $\kappa$ increases, the flux differential becomes inflected about $x_{1}=0$ [Fig. 3(a)]. We can think of this heuristically as follows. If the diffusive length scale is much shorter than the domain size $(\kappa \gg 1)$, then only particles initially created near the sink will fall into it. Therefore, the flux differential is only significantly nonzero close to the boundaries (or another sink). In essence, the geometry sensing of the system breaks down. On the other hand, when the diffusive length scale is much longer than the domain size $(\kappa \ll 1)$, particles can explore the entire domain before decaying and so the flux differential across the sink reflects its position on the domain, with the fluxes into the sink from either side balancing at mid-domain. One can also imagine that if strength of the sinks depended on the flux going into them, then this regime would lead to competition between sinks. The relevance to Turing systems will be made clear later.

We can make sink movement explicit by specifying the sink velocities. Given our results above, two natural choices are to take the sink velocities as either directly proportional to the flux differentials $\Delta J_{i}$,

$$
\frac{d x_{i}}{d t}=v \Delta J_{i}(\boldsymbol{x})
$$

or to the derivative of the mass $M(x)$ with respect to the sink positions

$$
\frac{d x_{i}}{d t}=-\frac{n}{2} \nu D \frac{\partial}{\partial x_{i}} M(\boldsymbol{x})
$$

where $v$ is some parameter and the choice of prefactor is for later convenience. For the latter choice, the system is analogous to that of $n$ overdamped particles moving in a potential $U(\boldsymbol{x}) / k_{B} T=\frac{n v}{2} M(\boldsymbol{x})$. Note also that while the velocities in (11) are specified in terms of local quantities, in (12), they are specified in terms of the global quantity $M(\boldsymbol{x})$.

In either case, the steady-state solution consists of regularly positioned sinks as this is the configuration for which the fluxes balance and for which the mass is at its unique minimum. This holds as long as $\delta>0$. For $\delta=0$, all the velocities (a)

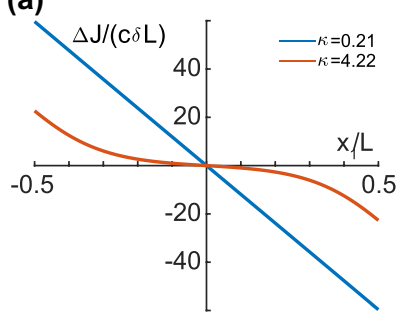

(c)

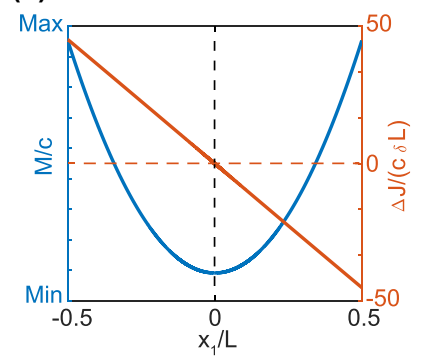

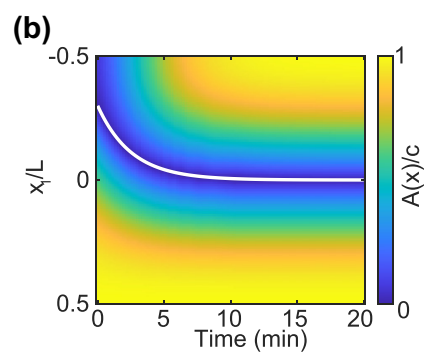

(d)

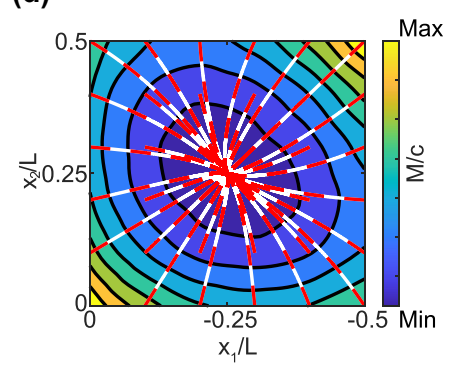

FIG. 3. Moving point sinks are regularly positioned and their movement depends on the diffusive length scale. (a) Flux differential across a point sink calculated analytically as a function of sink position $x_{1}$ for two values of $\kappa$. (b) When $\Delta J$ is linear in $x_{1}$ the sink moves exponentially to mid-domain. The flux differential across a point sink is linear in sink position for $\kappa \ll 1$. It vanishes at the middle of the domain. (c) Mass $M$ in system as a function of sink position for a single point sink is plotted in blue. The mass is minimal as the sink approaches the middle of the domain. (d) Sample trajectories of the two-sink system. White lines are sample trajectories obtained using (11), while the overlaid red dashed lines are trajectories obtained using (12). The colored contour shows the total mass $M$ as a shown as a function of the sink positions. The minimum occurs at the steadystate configuration (sinks at opposite quarter positions). Parameters: $D=0.3, \lambda=166.1, c=1, L=1, v=1 . \kappa=0.21$ in (b) and (c). In (d) $L=2$.

vanish identically. Hence, all sink positions are stable in that limit. This is the same behavior that we found in the previous section for the mass-conserved Turing system.

If we consider sinks moving on much slower timescale than that of diffusion, we can use the steady-state solution for $A(x)$ given in (4) to solve the dynamic system (11). We find, as expected, that a single sink moves exponentially to mid-domain [Fig. 3(b)]. We also find that increasing $\delta$, which increases the flux through the system, leads to faster sink movement (Fig. S2 [13]) reminiscent of the Turing system [Fig. 2(b)]. On the other hand, if we decrease $D$, which decreases the diffusive length scale without affecting the flux through the system, the sink moves more slowly towards mid-domain (Fig. S2 [13]). We also considered the system with two sinks and confirmed that the steady-state solution consists of quarter-positioned sinks, the configuration that minimizes the total mass of $A$ [Fig. 3(d)].

We can also use the steady-state solution of $A(x)$ to make explicit a correspondence between the two choices for the sink velocities. While the steady states of the two systems are identical, their dynamics are not in general the same. However, we found that (Appendix B) when $\delta$ is small (or in terms of the dimensionless parameters $\lambda \gg 1, \kappa \ll 1$ ), the 
two expressions become equivalent. This equivalence was apparent even for our default parameter set: the sink trajectories arising for either choice were almost identical [Fig. 3(d)].

\section{COMPARISON WITH THE TURING SYSTEM}

The similarity between moving point sinks (Fig. 3 ) and the movement of peaks in a Turing pattern (Figs. 1 and 2) is striking. It suggests that the movement and steady-state positions of peaks in a Turing pattern may be due to a dependence of the peak velocity on the flux differential (of the fast species across a peak of the slow species) or due to the total mass of the fast species acting (approximately) as a potential energy surface. Note that in the following we restrict ourselves to Turing patterns consisting only of interior peaks, as boundary peaks are not amenable to a point-sink approximation (see below). First, we introduce the following definition of the flux differential into the peak of a single-peak Turing pattern:

$$
\Delta J_{s}(t)=D_{u} \frac{\int_{-L / 2}^{L / 2} \frac{\partial u(x, t)}{\partial x} v(x, t) d x}{\int_{-L / 2}^{L / 2} v(x, t) d x} .
$$

If $v$ is proportional to a Dirac delta function, an approximation we will use below, this expression reduces to the flux differential of $u$, defined similarly to (7). We note that a similar expression has already been used to describe the flux into a spatial sink in the context of plasmid positioning [53]. We initialized the system with a single peak and monitored $\Delta J_{s}$ as a function of the peak position and velocity. We found that, like for point sinks [Fig. 3(c)], the flux differential is, away from the domain boundaries, directly proportional to the displacement from mid-domain [Fig. 4(a)]. Thus, peaks do indeed move with a velocity proportional to the flux differential.

However, it is not clear how to extend the definition of the flux differential to patterns with multiple peaks as well as to higher dimensions in which Turing patterns can consist of complex structures such as stripes, spirals, and hexagons. The concept of mass minimization on the other hand is easy to generalize. When we examined the total mass (concentration) of $u$ (the fast species) in the system, $M=\frac{1}{L} \int_{-\frac{L}{2}}^{\frac{L}{2}} u(x, t) d x$, we found that it decreases monotonically as the peak moves to mid-domain, modulo boundary effects [Fig. 4(a)]. Further, when we initialized the system with two peaks positioned at various locations, we found similar behavior [Fig. 4(c)] suggesting that, for a given number of peaks, the regularly positioned configuration minimizes the total mass of $u$, just as we have proven for point sinks in the previous section. Indeed, the trajectories show a remarkable similarity to those of moving point sinks [Fig. 3(d)].

To explore this analytically, we considered the singular limit $D_{v} \ll D_{u}$ in which the peaks in $v$ take the form of narrow spikes or pulses of width $\epsilon=\mathcal{O}\left(\sqrt{D_{v} / \gamma}\right)$ (Fig. S3 [13]). Away from the spike, $v$ is approximately constant with a value $v_{\text {out }}$ that is much smaller than $u$. This limit allows the use of nonlinear analysis methods to study the existence, stability, and dynamics of Turing patterns (see [42] for a review). Here, our goal is simply to derive an approximation for $u$ in this limit by treating the spikes of $v$ as Dirac delta functions as described below.

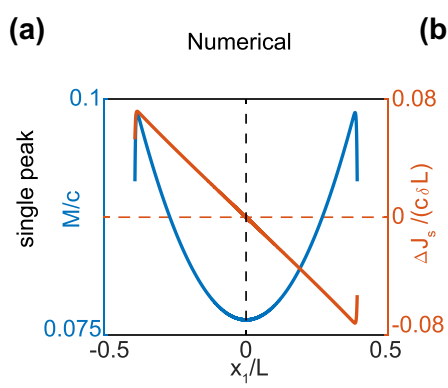

(b)

Analytical

(c)

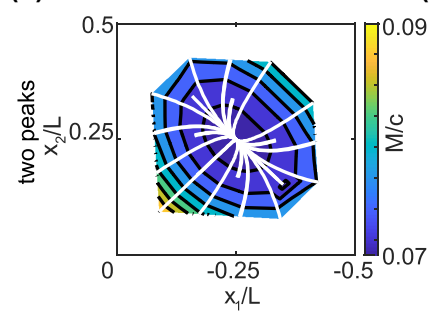

(d)
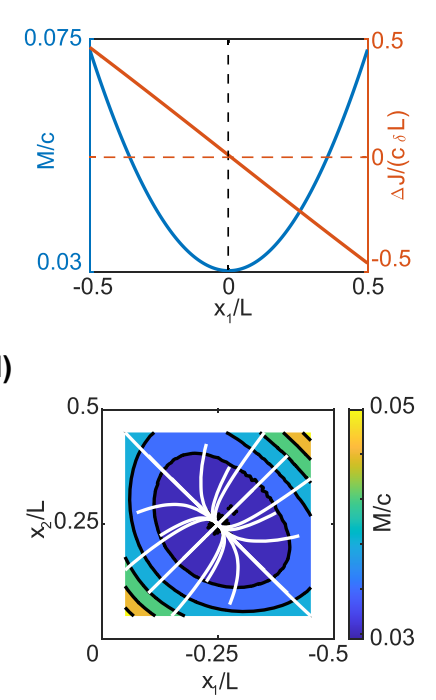

FIG. 4. The mass of $u$ is minimized at regular positions. (a) Flux differential measured numerically using (13) for a single spike (orange) is a linear function of the peak position. The mass of the fast species $M$ (blue) is minimized at mid-domain. See also Fig. S3 [13]. (b) The same quantities as in (a) but for the analytical expressions from the spike approximation [(21) and $\left.M=c-\hat{\rho}_{1+}^{\prime}\right]$. (d) Mass minimization for two peaks. Trajectories of two peaks as they move towards opposite quarter positions (white lines). The contours and color bar represent the mass $M$ interpolated from trajectories. The mass is minimized for regular positioning. (d) Same as (c) but trajectories obtained from the approximation of peaks as spikes using (22). Parameters: $L=2$ in (a) and (b), $L=4$ in (c) and (d); $D_{v}=0.0012$, otherwise default. This gives $\sigma=0.0146$ in (b) and $\sigma=0.0073$ in $(\mathrm{d})$.

We look for steady-state solutions consisting of $n$ spikes at positions $x_{1}, \ldots, x_{n}$. We assume that $u$ changes slowly within each spike and so can be approximated by a constant $u_{i}$ and within each spike $u_{i} \ll v$. First, we introduce the inner coordinate $y_{i}=\left(x-x_{i}\right) / \epsilon$ within each spike. We then have the following system for the inner variable $v_{i}(y)$ :

$$
\begin{array}{r}
\frac{D_{v}}{\epsilon^{2}} \frac{d^{2} v_{i}}{d y_{i}^{2}}+\beta u_{i} v_{i}^{2}-(\gamma+\delta) v_{i}=0, \\
v_{i} \rightarrow 0 \quad \text { as } \quad y_{i} \rightarrow \pm \infty,
\end{array}
$$

which gives

$$
v_{i}=\frac{3}{2} \frac{\gamma+\delta}{\beta u_{i}} \operatorname{sech}^{2}\left(\sqrt{\frac{\gamma+\delta}{D_{v}}} \frac{\epsilon y_{i}}{2}\right) .
$$

In the outer region, each spike is approximated by a weighted Dirac delta function and we therefore replace the $v$ and $u v^{2}$ terms by Dirac delta functions with weights $w_{1}$ and $w_{2}$ given by

$$
\begin{array}{r}
w_{i, 1}=\epsilon \int_{-\infty}^{\infty} v_{i}\left(y_{i}\right) d y_{i}=6 \frac{\sqrt{D_{v}(\gamma+\delta)}}{\beta u_{i}}, \\
w_{i, 2}=\epsilon u_{i} \int_{-\infty}^{\infty} v_{i}^{2}\left(y_{i}\right) d y_{i}=6 \frac{\sqrt{D_{v}}(\gamma+\delta)^{3 / 2}}{\beta^{2} u_{i}},
\end{array}
$$


respectively. Note that since $\mathcal{O}\left(w_{i, 1}\right)=1$ (each spike must have finite weight), we find that $\mathcal{O}\left(u_{i}\right)=\epsilon$ and therefore $\mathcal{O}\left(v_{i}\right)=\epsilon^{-1}$. Away from the spikes, $v$ is taken to be a constant $v_{\text {out }}$. Therefore, from (2b) we have

$$
\beta u\left(u+v_{\text {out }}\right)^{2}-(\gamma+\delta) v_{\text {out }}=0
$$

in the outer region. Given that $u$ and $v_{\text {out }}$ must both scale to leading order with a positive power of $\epsilon$ [due to the condition $\left.\frac{1}{L} \int_{-L / 2}^{L / 2}(u+v) d x=c\right]$, this equation implies that $\mathcal{O}\left(u^{3}\right)=$ $\mathcal{O}\left(v_{\text {out }}\right)$, i.e., in the spike limit $v_{\text {out }} \ll u$ in the outer region.

We obtain the outer equation for $u$ by replacing the $v$ terms at the spike by weighted delta functions:

$$
\begin{gathered}
D_{u} \frac{d^{2} u}{d x^{2}}-\sum_{i=1}^{n}\left[\beta\left(2 u_{i}^{2} w_{i, 1}+w_{i, 2}\right)-\gamma w_{i, 1}\right] \delta\left(x-x_{i}\right) \\
-\delta v_{\text {out }}+c \delta-\delta u=0
\end{gathered}
$$

where we used (14) to simplify the outer contribution. We can neglect the $u_{i}^{2}$ term since $\mathcal{O}\left(u_{i}\right)=\epsilon$ and the $v_{\text {out }}$ term since $\mathcal{O}\left(v_{\text {out }}\right)<\mathcal{O}(u)$ to arrive at

$$
D_{u} \frac{d^{2} u}{d x^{2}}+c \delta-\delta u-\sum_{i=1}^{n} \frac{\rho}{u} L \delta\left(x-x_{i}\right)=0
$$

with $\rho=6 \frac{\sqrt{D_{v}}}{L} \frac{\delta \sqrt{\gamma+\delta}}{\beta}$. Note the inverse dependence on $u$ in the point-sink term (which we call an inverted sink). This form is also obtained for other Turing systems with a $u v^{2}$ nonlinearity, such as the Schnakenberg and Brusselator models [35,54], as we show in Appendix C and the Supplemental Material [13], respectively.

Following the approach of the previous section, the solution to (16) is given by

$$
u(x)=c-\sum_{i} \rho_{i}^{\prime} G\left(x ; x_{i}\right),
$$

where the Green's function is defined as for point sinks but in terms of the corresponding dimensionless parameter $\kappa=$ $L \sqrt{\frac{\delta}{D_{u}}}$, the ratio of the length of the domain to the diffusive length scale of $u$ (henceforth, $\kappa$ replaces $b$ in the set of dimensionless parameters of the system). The coefficients $\rho_{i}^{\prime}=\rho_{i}^{\prime}(\boldsymbol{x})$ are now determined by the nonlinear algebraic system

$$
\rho_{i}^{\prime}=\sigma \frac{c^{2}}{u\left(x_{i}\right)}, \quad i=1, \ldots, n
$$

where $\sigma=\frac{\rho}{c^{2} \delta}=6 \frac{\sqrt{b+1}}{a \sqrt{\Gamma}}$ is the second dimensionless parameter of (16). The inverse dependence on $u\left(x_{i}\right)$ makes solving this algebraic system challenging. For a general choice of sink positions $x_{i}$, there are $n$ coupled quadratic equations in $\rho_{i}^{\prime}$, and therefore up to $2^{n}$ real solutions. However, this multiplicity of solutions collapses in the spike limit $\sigma \rightarrow 0$, in which the only physical solution is ${ }^{2}$

$$
\boldsymbol{\rho}^{\prime}=c \boldsymbol{G}^{-1} \hat{\boldsymbol{e}},
$$

\footnotetext{
${ }^{2}$ In the limit $\sigma \rightarrow 0,(18)$ becomes $\rho_{i}^{\prime}\left[c-\sum_{j} \rho_{j}^{\prime} G\left(x_{i} ; x_{j}\right)\right]=0$. Since taking any $\rho_{i}^{\prime}=0$ gives a solution of the system without the $i$ th spike, these are unphysical solutions.
}

where $\boldsymbol{G}_{i j}=G\left(x_{i} ; x_{j}\right)$ and $\hat{\boldsymbol{e}}$ is the column vector with all unit entries. This is precisely the same solution obtained in the perfect sink limit $\lambda \rightarrow \infty$ of the point-sink system [Eqs. (4) and (6)]. Thus, in the singular spike limit, steady-state solutions of the Turing system are equivalent to that of a system of perfect sinks, a surprising equivalence given the inverted prefactor in (16).

We next solve the system for a single arbitrarily positioned spike. We find two solutions corresponding to different spike amplitudes

$$
\frac{\rho_{1, \pm}^{\prime}}{c}=\frac{1 \pm \sqrt{1-4 \sigma G\left(x_{1} ; x_{1}\right)}}{2 G\left(x_{1} ; x_{1}\right)}
$$

and corresponding masses $M_{1, \pm}=c-\rho_{ \pm}^{\prime}$. Since we only ever observe spikes with large amplitudes, i.e., patterns in which almost all the mass of the system is contained within spikes, we assume that the low amplitude solution is unphysical for finite $\sigma$ and not only in the spike limit $\sigma \rightarrow 0$ (or unstable in the context of the time-dependent system, see below). Defining the flux differential across a spike analogously to (8),

$$
\Delta J_{i}(\boldsymbol{x})=-\frac{D_{u}}{2} \sum_{j} \rho_{j}^{\prime}\left[G_{x}\left(x_{i}^{+} ; x_{j}\right)+G_{x}\left(x_{i}^{-} ; x_{j}\right)\right],
$$

we find a linear dependence on the spike position in the regime $\kappa \ll 1$,

$$
\begin{aligned}
\frac{\Delta J_{1}}{c \delta L} & =-\frac{1}{2} \frac{\rho_{+}^{\prime}}{c} \frac{\sinh \left(2 \kappa \frac{x_{1}}{L}\right)}{\sinh (\kappa)} \\
& \approx-\frac{(1+\sqrt{1-4 \sigma})}{2} \frac{x_{1}}{L}+O\left(\kappa^{2}\right),
\end{aligned}
$$

consistent with our numerical observations [Figs. 4(a) and 4(b)] and just as we found for the noninverted sinks in the previous section [Fig. 3(c)]. Furthermore, in the spike limit $\sigma \rightarrow 0, \Delta J_{1}=c \delta x_{1}$ is linear in $\delta$, consistent with our numerical observations (for which $\sigma=0.0463$ ) [Fig. 2(b)]. We also find that $M_{1+}$, the total mass of $u$, is minimized at mid-domain [Fig. 4(b)]. However, while the mass and flux differential displayed very similar qualitative profiles [Fig. 4(b)], the agreement was not quantitative. In particular, the flux differentials disagree by an order of magnitude. This is likely because the analytic flux differential is defined at the interface region between the inner and outer solutions, which is where the approximation is least accurate. We will see in the next section that our main result is unaffected by this disparity.

The observation that peaks in a Turing pattern move with a velocity proportional to the flux differential across them [Figs. 2(a), 2(b), 4(a), and S3] suggests that the spike approximation can be extended to account for spike movement by specifying the spike velocities as

$$
\frac{d x_{i}}{d t}=v \Delta J_{i}(\boldsymbol{x})
$$

where $v$ is some unknown parameter. A similar expression has been obtained for the Brusselator model using particular singular limit in which the feed term is taken to zero with a scaling of $\epsilon^{1 / 2}$ [55]. By the correspondence with point sinks, this expression becomes equivalent in the spike $\sigma \rightarrow 0$ and long diffusive length scale and low-mass-flow regime $\kappa \rightarrow 0$ (or equivalently $\delta \rightarrow 0$ ) to a description in which the mass 
$M$ acts as potential and the sinks as overdamped particles as in (12). Together with (18), (22) defines a differentialalgebraic system for the dynamics of $n$ spikes. However, not all spike configurations are stable. Based on our numerical observations, stable solutions consist only of regularly positioned spikes of the same height $\left(\rho_{i}^{\prime}=\rho^{\prime}\right)$, also referred to as symmetric spike solutions, just as for the Turing system itself. Consistent with this, the flux differentials of these solutions vanish, $\Delta J_{i}(\overline{\boldsymbol{x}})=0$, via the properties of the Green's function, just as for the point sinks of the previous section (and Appendix A).

As an example, we consider the case of two spikes. For our parameter set the system then has up to four real solutions for each configuration $\left(x_{1}, x_{2}\right)$. In Fig. $4(d)$, we show sample trajectories [of the real solution branch with the smallest mass $M$; the other real solutions lead to very weak sinks and almost uniform $u(x)]$. The similarity to the numerical observation [Fig. 4(c)] and the system of point sinks in the previous section [Fig. 3(d)] is apparent. In both cases, the steady-state solution consists of quarter position peaks and spikes and minimizes the mass of $u$.

Let us summarize our results. We have shown that the movement and positioning of peaks in a Turing pattern is akin to that of a system of moving point sinks. First, the regular, periodic steady-state positions are a result of the flow (creation, diffusion, decay) of mass through the system, and not by some dominant linear mode. The steady-state configuration is the one for which all the flux differentials balance and this is also the configuration that minimizes the total mass of the fast species. We found empirically that the movement of peaks (in the slow species) is well described by the peak velocity being proportional to the flux differential of the fast species across it. Furthermore, in the spike limit, this is equivalent to the total mass of the fast species acting as a potential through which the peaks move as overdamped particles. While this does not imply that the mass or some other function acts as a potential away from this limit (i.e., that $\Delta \boldsymbol{J}$ is a conservative vector field in general), and indeed the trajectories specified by such a relationship are less consistent with the numerical results, the mass is nonetheless minimal at the steady-state configurations [Figs. 4(a) and 4(c)]. In the next section, we will see that we can use the steady-state mass to compare the "energy" of patterns with different numbers of peaks and in this way predict the preferred number of peaks at steady state, and not just their positions.

\section{COMPETITION AND PATTERN SELECTION}

We have seen that in the mass-conserved limit $\delta \rightarrow 0$, the model exhibits a complete coarsening effect in which the only stable patterns consist of a singe peak positioned somewhere in the interior of the domain [Fig. 2(d)] or, on a short domain, an interface. We have also seen that for small $\delta$ the model exhibits incomplete coarsening. With our default parameters (with $L=4$ ), linear stability predicts that mode $n=8$ (four peaks) will dominate [Fig. 1(a)]. While this is true initially, the pattern subsequently coarsens so that we most frequently obtain two peaks (dominated by mode $n=4$ ) [Figs. 1(b), 1(c), and S1D]. This coarsening effect is also referred to as a competition instability and has previously been studied in the context of spike solutions [35-37]. To our knowledge, there is currently no way to determine which pattern is finally obtained. Note that this is a narrower question than asking which patterns are stable since Turing systems are in general multistable.

This motivated us to explore the connection between coarsening and the flow rate $\delta$ in more detail. We measured the distribution of steady-state patterns obtained for different values of $\delta$ (through the dimensionless parameter $\kappa$ ) and compared against the prediction of linear instability [Figs. 5(a) and $5(\mathrm{~b})]$. We used periodic boundary conditions to avoid peaks on the boundary that are not described by the spike approximation. We found that for $\kappa \gtrsim 1$ linearly stability analysis correctly predicts the dominant mode at steady state. However, for $\kappa \lesssim 1$, a coarsening process occurs and the steady-state pattern is dominated by a lower mode than that predicted. Given our previous observations on the role of the diffusive length scale, we explain these results as follows. When the diffusive length scale is longer than the domain size, all peaks compete for $u$ molecules created across the domain. Whereas, when the length scale is short, peaks only absorb molecules of $u$ created within a distance given by the diffusive length scale and therefore compete less or not at all. Competition is also due to the fact that decreasing $\delta$ also decreases the total flux through the system $(c \delta L)$.

We next applied the spike approximation developed in the previous section. We decreased $D_{v}$ from the default value so that the obtained pattern was reasonably spike-like [Fig. 5(c)] while at the same time not resulting in a very much enlarged Turing space (since we want to sweep over different values of $\delta$ ). We considered only symmetric, regularly positioned spike solutions, which are the only observed steady-state solutions. For $n$ spikes, we obtain two possible values of $\rho^{\prime}$, of which we take the larger $\rho_{+}^{\prime}$ (the other corresponds to extremely weak spikes, i.e., $\rho_{-}^{\prime} \approx 0$ ). This gives a solution $u(x)=c-\rho_{+}^{\prime} \sum_{i} G\left(x ; \bar{x}_{i}\right)$ with mass

$$
M / c=1-n \frac{1+\sqrt{1-2 \kappa \sigma \operatorname{coth}\left(\frac{\kappa}{2 n}\right)}}{\kappa \operatorname{coth}\left(\frac{\kappa}{2 n}\right)} .
$$

Note that for a real solution we must have $1>2 \kappa \sigma \operatorname{coth}\left(\frac{\kappa}{2 n}\right)$. Therefore, for a given choice of parameters, there is an upper bound on the number of spikes that a solution can contain. In general, a solution exists for multiple values of $n$. However, numerically, we observe a very narrow distribution of the number of peaks [Figs. S1 [13] and 5(e)]. We hypothesized that mass minimization might play a role. Indeed, when we examined the mass $M$ of solutions consisting of different numbers of spikes at their respective steady-state positions, we found that the mass is minimal for a specific number of spikes [Fig. 5(d)]. This could also be seen by plotting the mass as a function of $\kappa$ for different values of $n$ (Fig. S4 [13]). The value of $n$ at the minimum decreases with $\kappa$, with a single spike being minimal at $\kappa \rightarrow 0$. The curves invert so that as $\kappa$ is increased multiple spikes produce the lowest mass. Given that we have already shown that the mass of $u(x)$ is minimal at the steady state, we hypothesized that it could also be used to compare solutions with different numbers of peaks and therefore identify a preferred "minimum energy" state. 
(a)

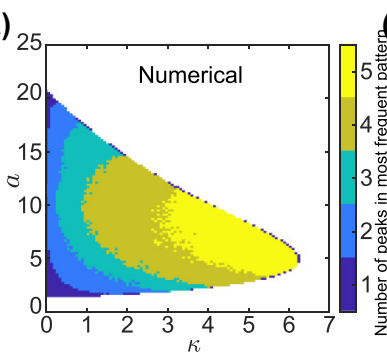

(c)
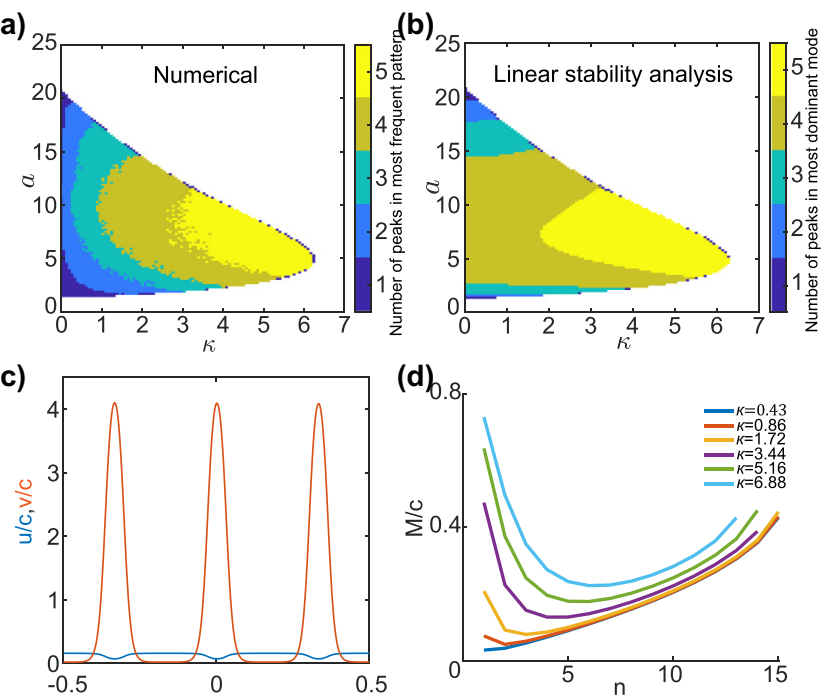

(d)

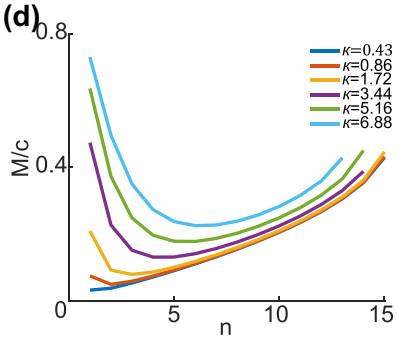

(e)

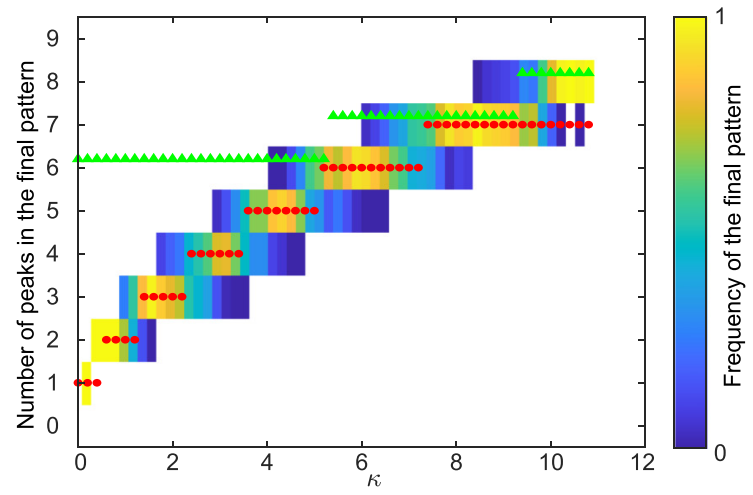

FIG. 5. Mass minimization predicts peak number in reaction diffusion systems. (a) The number of peaks in the most frequent steady-state pattern is plotted as a function of $a$ and $\kappa$. For each set of parameters, the most frequent pattern was obtained from five simulations each initialized with a different random perturbation from the uniform state. The simulations were run for long enough to ensure the steady-state pattern was reached. (b) The number of peaks in the mode with the greatest growth rate as predicted by linear stability analysis is plotted as a function of $a$ and $\kappa$. Plots (a) and (b) are similar for $\kappa \gg 1$. They disagree for $\kappa \leqslant 1$, which indicates coarsening. (c) Example of a steady-state pattern in the spiky limit. (d) Normalized total mass $M / c=1-n \rho_{+}^{\prime} / c$ plotted as a function of $n$ for different values of $\kappa$. There exists a critical $n$ for which the mass is minimal. (e) The numerically obtained distribution of peak number at steady state for different values of $\kappa$ (color scale) overlaid with the prediction of the dominant pattern from linear stability (green triangles) and the prediction from mass minimization (red circles). Mass minimization correctly predicts the number of peaks at steady state. Data from 50 simulations for each parameter set. Parameters: Default values as in Fig. 1 with $L=4$ except (c)-(e) which use $D_{v}=0.006$ (to make peaks narrower). See also Figs S4 and S5 [13].

We compared the number of spikes predicted by this mass minimization principle against the distribution of patterns obtained numerically (starting from a small random perturbation around the uniform state). We found remarkable agreement [Fig. 5(e), red circles]. Mass minimization correctly predicts the most frequent pattern obtained over the entire range of $\kappa$, including, most importantly, the regime in which coarsening occurs. There is significant deviation only at the transition points and close to exiting the Turing regime at high $\kappa$. In comparison, the linear prediction only agrees for the highest values of $\kappa$, i.e., close to onset [Fig. 5(e), green triangles]. Remarkably, the prediction was also reasonably accurate even when the solution is not very spikelike, as for our default parameter set (Fig. S5 [13]) and towards the boundary of the Turing space (Fig. 2). Thus, mass minimization not only explains where the peaks of a Turing pattern are positioned, but also how many peaks there will be at steady state, after any coarsening has taken place. Importantly, it does so far from onset and hence outside the region where weakly nonlinear approaches such as the amplitude equations are valid.

To investigate the general applicability of these results, we performed a similar analysis of the Brusselator model (Appendix C). This model also has the form of a mass-conserved model with additional production and decay terms and a dimensionless parameter $b$ characterizing the mass flow through the system (with $b=0$ being the mass-conserved limit). However, unlike the model in (2), the Brusselator does not have a length-scale parameter $\kappa$, rather the diffusive length scale is infinite, as determined from the form of its outer equation in the spike limit (Appendix C). Consistent with this, we observed that the Brusselator exhibits coarsening across the entire range of $b$ [Figs. 6(a) and 6(b)]. Following the same approach as above, we derived an expression for the total mass $M$ of the fast species of symmetric $n$-spike solutions

$$
M=\frac{6 n(b+1)^{3 / 2}}{a \sqrt{\Gamma}}+\frac{\Gamma b}{12 d(b+1) n^{2}} .
$$

This mass is again minimized for a particular number $n=n_{c}$ of spikes and we found this minimum to be an excellent predictor of the final pattern obtained after coarsening [Fig. 6(c)].

Finally, we examined a third system, the Schnakenberg model. Unlike the previous two, this model does not have a mass-conserved Turing system as a limit. Likely as a result of this, we find no evidence of a dynamic coarsening instability. Nevertheless, the final steady-state pattern is often not precisely that predicted by linear stability. Applying the spike approximation, we found that the outer equation of this model, and hence the expression for the mass of the fast species, has the same form as for the Brusselator (see Supplemental Material [13]). On comparing of the number of peaks predicted by minimizing the mass and by linear stability analysis, we found that while the two approaches were in broad agreement, mass minimization was the better predictor of the obtained pattern.

While we can not exclude the possibility that mass minimization is only predictive for patterns consisting of wellseparated peaks as studied here, the consistency of the results over three different systems nonetheless hints at a fundamental property of Turing systems and warrants further study.

\section{DISCUSSION}

One of the main challenges for the physics of pattern formation is the prediction of which pattern will be obtained, not only at onset, i.e., at entry into the parameter space giving patterns, but generically for any parameter values. While linear stability analysis can give a prediction for the dominant mode, nonlinear effects mean that it can be inaccurate. Furthermore, 
(a)

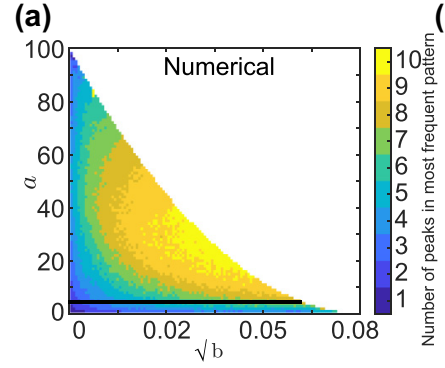

(b)

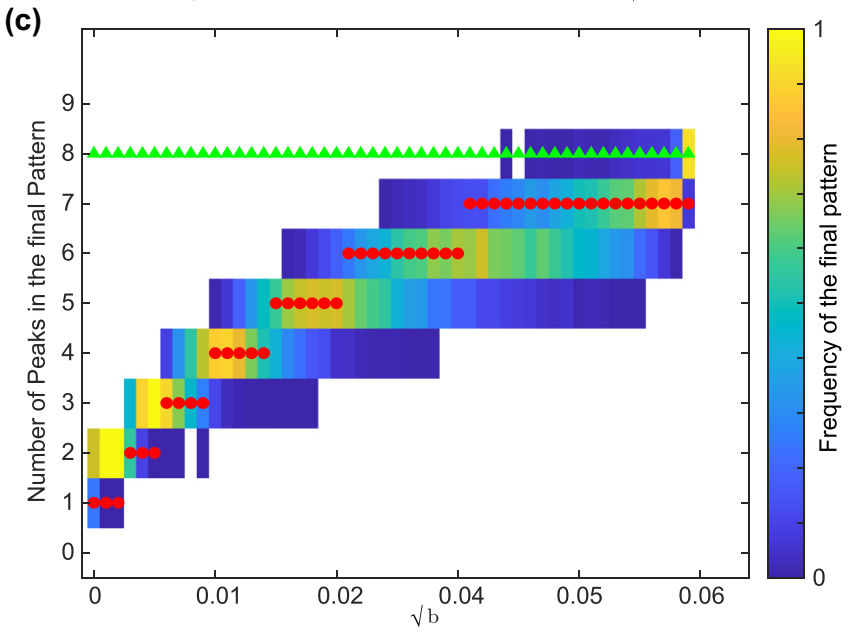

FIG. 6. Mass minimization correctly predicts the selected pattern after coarsening in the Brusselator. (a) The number of peaks in the most frequent steady-state pattern of the Brusselator model is plotted as a function of model parameters $a$ and $\sqrt{b}$ (see Appendix C). For each set of parameters, the most frequent pattern was obtained from 10 simulations each initialized with a different random perturbation from the uniform state. The simulations were run for long enough to ensure the steady-state pattern was reached. (b). The number of peaks in the mode with the greatest growth rate as predicted by linear stability analysis is plotted as a function of $a$ and $\sqrt{b}$. Coarsening observed over much of the range of $\sqrt{b}$ and the disparity with the linear stability prediction increase as $b \rightarrow 0$. Parameters: $d=100$ with $D_{u}=1, D_{v}=0.01$, and $\Gamma=15000$. (c) As Fig. 5(e), but for the Brusselator model. The numerically obtained distribution of the number of peaks at steady state for different values of $\sqrt{b}$ (color scale) is overlaid with the prediction of the dominant pattern from linear stability (green triangles) and the prediction from minimization of the mass of the fast species (24) (red circles). Mass minimization again correctly predicts the number of peaks at steady state. Data from 500 simulations for each parameter set. Black lines in (a) and (b) show the range of $\sqrt{b}$ values used within the $(a, \sqrt{b})$ Turing space. Parameters: $d=100, a=3.75, \Gamma=15000$.

as we have argued, linear analysis cannot explain the periodic nature of final patterns nor (with reflective boundary conditions) the positioning of peaks within the domain, which occurs dynamically in several settings that are outside of the linear regime (e.g., domain growth, coarsening, initialized peaks). Weakly nonlinear approaches, such as the method of amplitude equations, do exist but they are much less useful far from onset and while a recent phase-space approach has been introduced to study steady-state patterns in two-component mass-conserved systems [34,56,57], we still lack a general theory of pattern dynamics and selection far from onset.
Here, we have presented evidence that the flow of mass through the system is responsible for movement and regular positioning of peaks in a Turing pattern. We also showed that the number and positions of peaks at steady state are such that the mass of the fast species is minimized. This simple principle correctly predicted the preferred steady-state pattern for both our model and the Brusselator, even far from onset and in the presence of coarsening. Our expectation is that this will guide the development of new nonlinear approaches for the study of pattern selection, far from onset.

The insight came from analyzing the behavior of a diffusive system consisting of point sinks that move with a velocity proportional to the gradient. We showed that the flow of mass through such a system leads to sinks being positioned symmetrically and evenly across the domain (regularly positioned), as this is the unique configuration for which the gradient across each sink vanishes. We also showed that this configuration uniquely minimizes the total mass in the system. Consistent with this, we showed that in the low-mass-flow regime, the total mass acts as a potential and the sinks as overdamped particles.

We found that in the Turing system, peaks (of the slowly diffusing species $v$ ) also move toward the regularly positioned configuration with the same dynamics as point sinks and at a rate directly proportional to the rate of mass flow through the system. In doing so, the system minimizes the total mass of the fast species $u$. In the singular limit $D_{v} \rightarrow 0$, in which the peaks of the Turing pattern become narrow pointlike spikes [42], an analytical approximation showed that $u$ is indeed described by diffusion in the presence of point sinks but where the Dirac delta function terms have a $1 / u$ prefactor. Nevertheless, in the singular limit, solutions are that of a system of perfect sinks. However, for $D_{v}>0$ the "inverted" sink term leads to the total mass of $u$ having a nontrivial dependence on the number of spikes and the rate of mass flow through the system. As a result, there are a critical number of spikes (and hence wavelength) that minimize the mass of $u$. We therefore hypothesized that the mass of $u$, the fast species, could act, at least approximately, as a multiwell potential, i.e., that it could be used to assign an energy to patterns with different numbers of peaks and thereby identify the preferred steady state. In particular, we asked whether it could predict the steady-state pattern selected after the coarsening that occurs in our model. We found that this "mass minimization" principle could indeed predict, almost perfectly, the obtained patterns [Fig. 5(e)] and we confirmed this for another Turing system, the Brusselator (Fig. 6). It also gave a better prediction of the dominant pattern for the Schnakenberg model, which does not exhibit coarsening (see Supplemental Material [13]). Note that we are referring to which pattern is selected and not which patterns are stable. While the latter has already been studied for spike and mesa solutions (see [42] for a review), to our knowledge much less is unknown about the former.

The two models with coarsening have a parameter $\delta$ controlling the flow of mass through the system and the degree of coarsening. In the mass-conserved limit $\delta=0$, they display complete coarsening down to single peak, a seemingly generic property of two-component mass-conserved reaction diffusion systems that has been proved explicitly for several systems $[30-33,38,58,59]$. This complete coarsening is 
correctly predicted by mass minimization; in both models for $\delta=0$, the mass of the fast species is minimal for a single peak.

We speculate that complete coarsening is due to the absence of mass flow through the system rather than mass conservation per se. Consistent with this, the feed and decay terms in our model and in the Brusselator can be replaced with linear couplings to a sufficiently well-mixed third species, i.e., we can embed the open system inside a larger closed system. The result is a three-component mass-conserved model with the same pattern-forming behavior, including partial (not complete) coarsening. Indeed, we previously investigated a model with precisely this form [12]. While the entire threecomponent system exhibits mass conservation, there is still mass flow through the "subsystem" of the original two variables, the rate of which controls the degree of coarsening.

Are our results applicable to other systems? The outer equation for $u$ obtained in the singular limit $D_{v} \ll D_{u}[(16)]$ has the same form in other systems of the substrate-depletion type such as the Brusselator [35] (Appendix C), Schnakenberg (supplemental text) [54], and Gray-Scott [36,60] models and indeed all these models exhibit the same peak movement towards the regularly positioned configuration (see Supplemental Material [13]). Substrate-inhibition models that have peaks of the two species overlapping, such as that of Gierer and Meinhardt [61], also exhibit peak movement towards regular positions. However, the outer equation of these models have a point source term rather than a point sink [62]. The effect of this on mass minimization remains to be tested.

Might any of our results apply outside the regime of wellseparated peaks? That is currently unclear and testing it would require an analytical description of other solution types. We leave this for future work. However, the following observation suggests to us that mass minimization of the fast species may have some broader relevance. If the final pattern is selected according to minimizing the mass of the fast species, then that mass must be minimizable. We can therefore ask what would happen in a model in which the mass of the fast species is a fixed constant at steady state? Consider the following class of systems:

$$
\begin{aligned}
& \partial_{t} u=D_{u} \partial_{x}^{2} u-f(u, v)+a-u, \\
& \partial_{t} v=D_{v} \partial_{x}^{2} v+f(u, v)+b
\end{aligned}
$$

with $D_{v}<D_{u}$ and reflective or periodic boundary conditions. Note that at steady state the mass of $u$ is fixed. It is straightforward to show (see Supplemental Material [13]) that a system of this form cannot admit a Turing instability for any $f$. This result holds even if we replace $u$ in the last term by any function $g(u)$ with $g^{\prime}(u)>0$ at the homogeneous fixed point, and a corresponding measure $\int g(u) d x$ for the mass of $u$. There is no such restriction if the decay term is placed in the equation for $v$ (like for the Brusselator and Schnakenberg models) or in both equations [as in (2) and the Gray-Scott model]. Thus, for this general class of models, a Turing instability requires that the mass of the fast species must not be generically a fixed constant at steady state. Equivalently stated, systems in which mass leaves the system through only the fast species cannot exhibit a Turing instability. While this is a requirement for the applicability of a mass-minimization principle, the connection, if any, between pattern selection and stability of the base state is unclear. It may be that for some class of models, spatial instability of the base state is equivalent to the existence of a stable spatial solution for which the mass of the fast species is lower. Further study is required to investigate these questions.

\section{A. Numerical methods}

The simulations were performed on a spatial lattice $x \in$ $\left[-\frac{L}{2}, \frac{L}{2}\right]$ and time domain $t \in[0, T]$, where $L$ is the length of the spatial domain and $T$ the total time. The MATLAB solver pdepe was used to solve the time-dependent equation (2). The simulations were performed with the following default parameters (unless explicitly stated otherwise):

$$
\begin{gathered}
D_{u}=0.3, D_{v}=0.012, L=2, c=300, \\
\beta=1.5 \times 10^{-4}, \gamma=3.6, \delta=0.014 .
\end{gathered}
$$

The equivalent dimensionless parameters are

$$
d=25, a=3.75, b=0.0039, \Gamma=1200 .
$$

The simulations were run long enough so as to obtain the true steady state. The relative and absolute tolerances in the difference between two values of iteration were set to $10^{-6}$ and $10^{-12}$, respectively. We used reflective boundary conditions

$$
\left.\frac{\partial u}{\partial x}\right|_{x=-L / 2, L / 2}=\left.\frac{\partial v}{\partial x}\right|_{x=-L / 2, L / 2}=0,
$$

except for Figs. 5 and 6 and Figs. S5 and S7 [13], where we use periodic boundary conditions

$$
\begin{aligned}
& \left.u\right|_{x=-L / 2}=\left.u\right|_{x=L / 2},\left.\quad u^{\prime}\right|_{x=-L / 2}=\left.u^{\prime}\right|_{x=L / 2}, \\
& \left.v\right|_{x=-L / 2}=\left.v\right|_{x=L / 2},\left.\quad v^{\prime}\right|_{x=-L / 2}=\left.v^{\prime}\right|_{x=L / 2} .
\end{aligned}
$$

As the pdepe does not support such boundary conditions, we used the package Periodic Reaction Diffusion PDE solver [63]. The initial conditions were taken to be a random perturbation around the homogeneous steady state (drawn from a normal distribution with standard deviation of $1 \%$ ).

The differential algebraic systems (11), (12), and (22) were solved using the ode $15 \mathrm{~s}$ solver.

\section{B. Numerical comparisons for Fig. 4}

To compare the movement of peaks in the simulations with the analytical calculations (spike approximation), we initialize the peaks at different positions (by translation of the steady-state pattern) and monitor the evolution of the system. In Fig. 4(a) (single peak), we calculate the mass of the fast species $M(t)=\int_{-L / 2}^{L / 2} u(x, t) d x$, and the flux on the peak $\Delta J_{s}[(13)]$, at each time step as the peak approaches mid-domain. In Fig. 4(c) (two peaks), we generated the contours of $M$ by simulating the Turing system with 200 different initial peak positions $\left(x_{1}, x_{2}\right)$ (some of which are overlayed in white) and interpolating over the trajectories. Note that it is not clear how to define the flux differential across each peak in the case of more than one peak. Similarly, in Fig. 4(d), we solved the nonlinear algebraic system in (18) for different sink positions $\left(x_{1}, x_{2}\right)$. We obtain at most four different solutions of which we take the one with the lowest total mass $M$. Several analytical trajectories were obtained by solving the differential-algebraic system in MATLAB consisting of 
Eqs. (18) and (22), initialized with the solution having the lowest mass.

\section{APPENDIX A: POINT SINKS, FLUX BALANCE, AND MASS MINIMIZATION}

In this Appendix, we prove an important result described in the main text. The solution to (3) is given by

$$
A(x)=c-\sum_{i} \mu_{i}^{\prime} G\left(x ; x_{i}\right)
$$

where the Green's function is defined in (5) and the $\mu_{i}^{\prime}=$ $\mu_{i}^{\prime}(\boldsymbol{x})$ are determined by the algebraic equations

$$
\mu_{i}^{\prime}=\lambda\left(c-\sum_{j} \mu_{j}^{\prime} G\left(x_{i} ; x_{j}\right)\right)
$$

\section{Properties of the Green's function}

The explicit form of the Green's function is

$$
G\left(x ; x_{i}\right)=\frac{\kappa}{2} \frac{\cosh \left(\kappa \frac{x+x_{i}}{L}\right)+\cosh \left(\kappa \frac{\left|x-x_{i}\right|-L}{L}\right)}{\sinh (\kappa)} .
$$

The derivative of $G\left(x ; x_{i}\right)$ with respect to $x$ is discontinuous at $x=x_{i}$ :

$$
G_{x}\left(x ; x_{i}\right)= \begin{cases}\frac{\kappa^{2}}{2 L} \frac{\sinh \left(\kappa \frac{x+x_{i}}{L}\right)-\sinh \left(\kappa \frac{x_{i}-x-L}{L}\right)}{\sinh (\kappa)}, & -\frac{L}{2} \leqslant x<x_{i} \\ \frac{\kappa^{2}}{2 L} \frac{\sinh \left(\kappa \frac{x+x_{i}}{L}\right)+\sinh \left(\kappa \frac{x-x_{i}-L}{L}\right)}{\sinh (\kappa)}, & x_{i}<x \leqslant \frac{L}{2}\end{cases}
$$

$$
\begin{aligned}
\sum_{j} G_{i j} & =\frac{\kappa}{2 \sinh (\kappa)}\left[\sum_{j=1}^{n} \cosh \left(\kappa \frac{\bar{x}_{i}+\bar{x}_{j}}{L}\right)+\sum_{j=1}^{i} \cosh \left(\kappa \frac{\bar{x}_{i}-\bar{x}_{j}-L}{L}\right)+\sum_{j=i+1}^{n} \cosh \left(\kappa \frac{\bar{x}_{j}-\bar{x}_{i}-L}{L}\right)\right] \\
& =\frac{\kappa}{2 \sinh (\kappa)}\left[\sum_{j=1}^{n} \cosh [a(i+j-1-n)]+\sum_{j=1}^{i} \cosh [a(i-j-n)]+\sum_{j=i+1}^{n} \cosh [a(j-i-n)]\right] \\
& =\frac{\kappa}{2} \operatorname{coth}\left(\frac{\kappa}{2 n}\right) \quad \forall i,
\end{aligned}
$$

where $a=\frac{\kappa}{n}$ and the last step follows from the identity

$$
\sum_{j=1}^{n} \cosh [a(j+m)]=\operatorname{csch}\left(\frac{a}{2}\right) \sinh \left(\frac{a n}{2}\right) \cosh \left(\frac{a}{2}(2 m+n+1)\right) .
$$

We can similarly define a matrix $G_{x}^{+}$by evaluating the derivative of the Green's function at regular positioning $\left(G_{x}^{+}\right)_{i j}=$ $G_{x}\left(\bar{x}_{i}^{+} ; \bar{x}_{j}\right)$. Summing over the $j$ th column we find

$$
\sum_{j}\left(G_{x}^{+}\right)_{i j}=-\frac{\kappa^{2}}{2 L}
$$

in this case by using the identity

$$
\sum_{j=1}^{n} \sinh [a(j+m)]=\operatorname{csch}\left(\frac{a}{2}\right) \sinh \left(\frac{a n}{2}\right) \sinh \left(\frac{a}{2}(2 m+n+1)\right) .
$$

\section{b. Property II}

Since the summation of $G$ over any of its rows or columns is the same, the vector of $1 s, \hat{\boldsymbol{e}}$, is an eigenvector of $G$. Evaluating the defining equations for the $\boldsymbol{\mu}^{\prime}$, (A2), at regular positioning $\boldsymbol{x}=\overline{\boldsymbol{x}}$, we obtain the matrix equation

$$
(\lambda G+1) \mu^{\prime}(\overline{\boldsymbol{x}})=\lambda c \hat{\boldsymbol{e}}
$$


Since $\hat{\boldsymbol{e}}$ is an eigenvector of $\lambda G+1$, we must have that

$$
\boldsymbol{\mu}^{\prime}(\overline{\boldsymbol{x}})=C_{1} \hat{\boldsymbol{e}},
$$

i.e., all the $\mu_{i}^{\prime}$ are identical at regular positioning or, in other words, the profile of $A$ is symmetric. We can sum over any row and use (A7) to find

$$
\mu_{i}^{\prime}(\overline{\boldsymbol{x}})=\frac{\lambda c}{1+\lambda \frac{\kappa}{2} \operatorname{coth}\left(\frac{\kappa}{2 n}\right)}
$$

\section{Regular positioning and flux balance}

The flux differential across each sink is given by

$$
\Delta J_{i}=-D \sum_{j} \mu_{j}^{\prime}\left[G_{x}\left(x_{i}^{+} ; x_{j}\right)+\frac{\kappa^{2}}{2 L} \delta_{i j}\right] .
$$

We evaluate this expression for regularly positioned sinks $\boldsymbol{x}=\overline{\boldsymbol{x}}$. First we know from (A10) that all $\mu_{j}^{\prime}$ are identical for regularly positioned sinks. Then, from (A8), it follows that immediately that the flux differentials vanish at regular positioning

$$
\Delta J_{i}(\overline{\boldsymbol{x}})=0 \quad \forall i .
$$

We can show that this is the unique $\kappa$-independent configuration for which the flux differentials vanish by performing a power series expansion of $\Delta J_{i}$ in $\kappa$. Since for any $\kappa$ independent zero of $\Delta J_{i}(\boldsymbol{x})$ all terms in the $\kappa$ expansion must vanish independently, it suffices to show uniqueness for the $\kappa^{0}$ term. We first expand $\mu_{i}^{\prime}$ and $G^{\prime}\left(x_{i} ; x_{j}\right)$ :

$$
\begin{aligned}
\mu_{i}^{\prime} & =\mu_{0 i}+\mu_{2 i} \kappa^{2}+\cdots, \\
G\left(x_{i} ; x_{j}\right) & =G_{0}\left(x_{i} ; x_{j}\right)+G_{2}\left(x_{i} ; x_{j}\right) \kappa^{2}+\cdots .
\end{aligned}
$$

For the lowest order terms, we find first that $G_{0}\left(x_{i} ; x_{j}\right)=1$. Inserting this into the defining equation for the $\mu_{i}^{\prime}[(\mathrm{A} 2)]$, we have

$$
\mu_{0 i}^{\prime}=\left(c-\sum_{j} \mu_{0 j}^{\prime}\right) \lambda \quad \forall i
$$

which has solution

$$
\mu_{0 i}^{\prime}=\mu_{0}^{\prime}=\frac{\lambda c}{1+n \lambda} .
$$

We then have

$$
\begin{aligned}
\frac{\Delta J_{i}}{\delta L} & =-\frac{D}{\delta L} \sum_{j} \mu_{j}^{\prime}\left[G_{x}\left(x_{i}^{+} ; x_{j}\right)+\frac{\kappa^{2}}{2 L} \delta_{i j}\right] \\
& =-\frac{1}{2}\left[\sum_{j=1}^{n} \mu_{j}^{\prime} \frac{\sinh \left(\kappa \frac{x_{i}+x_{j}}{L}\right)}{\sinh (\kappa)}+\sum_{j=1}^{i} \mu_{j}^{\prime} \frac{\sinh \left(\kappa \frac{x_{i}-x_{j}-L}{L}\right)}{\sinh (\kappa)}-\sum_{j=i+1}^{n} \mu_{j}^{\prime} \frac{\sinh \left(\kappa^{\left.\frac{x_{j}-x_{i}-L}{L}\right)}\right.}{\sinh (\kappa)}+\mu_{i}^{\prime}\right] \\
& =-\frac{\mu_{0}^{\prime}}{2 L}\left[\sum_{j=1}^{n}\left(x_{i}+x_{j}\right)+\sum_{j=1}^{i-1}\left(x_{i}-x_{j}-L\right)-\sum_{j=i+1}^{n}\left(x_{j}-x_{i}-L\right)\right]+O\left(\kappa^{2}\right) \\
& =-\frac{n \mu_{0}^{\prime}}{L}\left[x_{i}-\frac{L}{n} i+\frac{L}{2}\left(\frac{1}{n}+1\right)\right]+O\left(\kappa^{2}\right) .
\end{aligned}
$$

Hence, all the flux differentials $\Delta J_{i}$ vanish for regularly positioned sinks $x_{i}=\bar{x}_{i}=\frac{L}{n} i-\frac{L}{2}\left(\frac{1}{n}+1\right)$ and this is the only configuration to do so for all $\kappa$. We assume, based on our numerical observations, that there are no additional $\kappa$-dependent states. Therefore, if we add time dependence to the system by specifying the sink velocities as being proportional to the their flux differential

$$
\frac{d x_{i}}{d t}=v \Delta J_{i}(\boldsymbol{x})
$$

then regularly positioned sinks is the unique fixed point of the resultant dynamical system [specified by the differentialalgebraic system defined by Eqs. (A2), (A5), and (A15).

\section{Regular positioning and mass minimization}

The total mass (or rather concentration) of $A$ is readily given by integrating Eq. (A1):

$$
M(\boldsymbol{x})=\frac{1}{L} \int_{-\frac{L}{2}}^{\frac{L}{2}} A(x) d x=c-\sum_{i=1}^{n} \mu_{i}^{\prime} .
$$

We would like to show that the regularly positioned configuration is the unique $\kappa$-independent stationary point of $M$. First, we will show that

$$
\left.\frac{\partial}{\partial x_{m}} M\right|_{x=\bar{x}}=-\left.\frac{\partial}{\partial x_{m}} \sum_{i} \mu_{i}^{\prime}\right|_{x=\bar{x}}=0 .
$$

Using (A2) $\left(\mu_{i}^{\prime}=\lambda\left[c-\sum_{j} \mu_{j}^{\prime} G\left(x_{i} ; x_{j}\right)\right]\right)$, we can evaluate the derivative of $\sum_{i} \mu_{i}^{\prime}$ with respect to an arbitrary sink position $x_{m}$ :

$$
\frac{\partial}{\partial x_{m}} \sum_{i} \mu_{i}^{\prime}=-\lambda \sum_{i, j} \mu_{j}^{\prime} G_{x_{m}}\left(x_{i} ; x_{j}\right)-\lambda \sum_{i, j} G\left(x_{i} ; x_{j}\right) \frac{\partial}{\partial x_{m}} \mu_{j}^{\prime} .
$$

Evaluating this expression at regular positioning, and defining $C:=\sum_{j} G\left(\bar{x}_{i} ; \bar{x}_{j}\right)=\frac{\kappa}{2} \operatorname{coth}\left(\frac{\kappa}{2 n}\right)$ from (A7), we obtain

$$
\left.\left(\frac{1}{\lambda}+C\right) \frac{\partial}{\partial x_{m}} \sum_{i} \mu_{i}^{\prime}\right|_{\boldsymbol{x}=\overline{\boldsymbol{x}}}=-\left.\sum_{i, j} \mu_{j}^{\prime} G_{x_{m}}\left(x_{i} ; x_{j}\right)\right|_{\boldsymbol{x}=\overline{\boldsymbol{x}}} .
$$


We have already seen in (A10) that all the $\mu_{j}^{\prime}$ are identical at regular positioning. Hence, we need only evaluate $\left.\sum_{i, j} G_{x_{m}}\left(x_{i} ; x_{j}\right)\right|_{x=\bar{x}}$. Inserting the definition of $G\left(x_{i} ; x_{j}\right)$ from (A3) we have

$$
\begin{aligned}
\left.\sum_{i, j} G_{x_{m}}\left(x_{i} ; x_{j}\right)\right|_{x=\bar{x}}= & \left.\frac{\kappa}{2 \sinh (\kappa)} \sum_{i, j} \frac{\partial}{\partial x_{m}}\left[\cosh \left(\kappa \frac{x_{i}+x_{j}}{L}\right)+\cosh \left(\kappa \frac{\left|x_{i}-x_{j}\right|-L}{L}\right)\right]\right|_{x=\bar{x}} \\
= & \frac{\kappa^{2}}{2 L \sinh (\kappa)} \sum_{i, j}\left\{\delta_{m i}\left[\sinh \left(\kappa \frac{x_{i}+x_{j}}{L}\right)+\operatorname{sgn}\left(x_{i}-x_{j}\right) \sinh \left(\kappa \frac{\left|x_{i}-x_{j}\right|-L}{L}\right)\right]\right. \\
& \left.+\delta_{m j}\left[\sinh \left(\kappa \frac{x_{i}+x_{j}}{L}\right)-\operatorname{sgn}\left(x_{i}-x_{j}\right) \sinh \left(\kappa \frac{\left|x_{i}-x_{j}\right|-L}{L}\right)\right]\right\}\left.\right|_{x=\bar{x}} \\
= & \left.\frac{\kappa^{2}}{L \sinh (\kappa)} \sum_{i}\left[\sinh \left(\kappa \frac{x_{m}+x_{i}}{L}\right)+\operatorname{sgn}\left(x_{m}-x_{i}\right) \sinh \left(\kappa \frac{\left|x_{m}-x_{i}\right|-L}{L}\right)\right]\right|_{x=\bar{x}} \\
= & \frac{\kappa^{2}}{L \sinh (\kappa)}\left[\sum_{i=1}^{n} \sinh [a(m+i-n-1)]+\sum_{i=1}^{m-1} \sinh [a(m-i-n)]-\sum_{i=m+1}^{n} \sinh [a(i-m-n)]\right. \\
= & \frac{\kappa^{2}}{L \sinh (\kappa)}[-\sinh (\kappa)-\sinh (-\kappa)]=0,
\end{aligned}
$$

where the last line follows from noting that the summations are the same as in (A8) but without the $i=m$ term. We have therefore shown that regular positioning is a stationary configuration of the total mass

$$
\left.\frac{\partial}{\partial x_{m}} M\right|_{x=\bar{x}}=0
$$

To show that regular positioning is the unique $\kappa$-independent stationary point, we proceed as in the previous section and perform a power series expansion of $M$ :

$$
M=M_{0}+M_{2} \kappa^{2}+\cdots
$$

It then suffices to show uniqueness for the first nontrivial order in the expansion. For the Green's function we have

$$
G_{0}\left(x_{i} ; x_{j}\right)=1, \quad G_{2}\left(x_{i} ; x_{j}\right)=\frac{x_{i}^{2}+x_{j}^{2}-L\left|x_{i}-x_{j}\right|}{2 L^{2}}+\frac{1}{12} .
$$

We already saw that $\mu_{0 i}^{\prime}=\mu_{0}^{\prime}=\frac{\lambda c}{1+n \lambda}$ and hence $M_{0}$ is a constant. Inserting these into the equation for $\mu_{2 i}^{\prime}$ using (A2),

$$
\mu_{2 i}^{\prime}=-\lambda \sum_{j}\left[\mu_{2 j}^{\prime}+\mu_{0 j}^{\prime} G_{2}\left(x_{i} ; x_{j}\right)\right]
$$

we obtain

$$
M_{2}=-\sum_{i} \mu_{2 i}^{\prime}=\frac{\lambda}{1+n \lambda} \mu_{0}^{\prime} \sum_{i} \sum_{j}\left(\frac{x_{i}^{2}+x_{j}^{2}-L\left|x_{i}-x_{j}\right|}{2 L^{2}}+\frac{1}{12}\right) .
$$

The derivative of $M_{2}$ is then proportional to

$$
\begin{aligned}
\frac{\partial}{\partial x_{m}} & \sum_{i} \sum_{j}\left[x_{i}^{2}+x_{j}^{2}-L\left|x_{i}-x_{j}\right|\right] \\
& =\frac{\partial}{\partial x_{m}}\left[2 n \sum_{i} x_{i}^{2}-\sum_{i} \sum_{j \leqslant i}\left(x_{i}-x_{j}\right) L-\sum_{i} \sum_{j>i}\left(x_{j}-x_{i}\right) L\right] \\
& =\frac{\partial}{\partial x_{m}}\left[2 n \sum_{i} x_{i}^{2}-L\left(\sum_{i} i x_{i}-\sum_{i}(n-i) x_{i}-\sum_{i} \sum_{j \leqslant i} x_{j}+\sum_{i} \sum_{j>i} x_{j}\right)\right] \\
& =\left[4 n x_{m}-L(m-(n-m)-(n-m+1)+m-1)\right]=4 n\left[x_{m}-\frac{L}{n} m+\frac{L}{2}+\frac{L}{2 n}\right]
\end{aligned}
$$


which vanishes only for the regularly positioned configuration

$$
x_{m}=\frac{L}{n} m-\frac{L}{2}\left(\frac{1}{n}+1\right)
$$

Hence, we have shown that regularly positioned sinks are the unique $\kappa$-independent configuration for which the total mass $M$ is stationary. Based on our numerical results, we assume that this configuration is generically a minimum.

\section{APPENDIX B: THE MASS ACTS AS A POTENTIAL AS $\delta \rightarrow 0$}

\section{Dynamics of a single point sink}

We consider the regime in which the timescale of gradient formation is much faster than that of sink movement. The profile $A(x)$ can therefore be approximated by (A1). The flux differential across a single sink is given by (A11):

$$
\begin{aligned}
\Delta J_{1} & =-D \mu_{1}^{\prime}\left[G_{x}\left(x_{1}^{+} ; x_{1}\right)+\frac{\kappa^{2}}{2 L}\right] \\
& =-D c \frac{\lambda}{\lambda G\left(x_{1} ; x_{1}\right)+1}\left[\frac{\kappa^{2} \sinh \left(2 \kappa x_{1} / L\right)}{2 L \sinh (\kappa)}\right],
\end{aligned}
$$

where

$$
\begin{aligned}
\mu_{1}^{\prime} & =\frac{\lambda c}{\lambda G\left(x_{1} ; x_{1}\right)+1}, \\
G\left(x_{1} ; x_{1}\right) & =\frac{\kappa}{2}\left[\frac{\cosh \left(\frac{2 \kappa x_{1}}{L}\right)}{\sinh (\kappa)}+\operatorname{coth}(\kappa)\right] .
\end{aligned}
$$

Now consider the derivative of the total mass (A16):

$$
\begin{aligned}
\frac{\partial M}{\partial x_{1}} & =\frac{d}{d x_{1}}\left(c-\mu_{1}^{\prime}\right)=-\frac{d}{d x_{1}} \mu_{1}^{\prime} \\
& =-c\left(\frac{\lambda}{\lambda G\left(x_{1} ; x_{1}\right)+1}\right)^{2} \frac{\kappa^{2} \sinh \left(2 \kappa x_{1} / L\right)}{L \sinh (\kappa)} .
\end{aligned}
$$

In the regime $\kappa \ll 1$ we can easily see that $G\left(x_{1} ; x_{1}\right)=1$. If sinks are also much stronger than the background decay rate, i.e., $\lambda \gg 1$, then we obtain the following relation:

$$
\Delta J=-\frac{D}{2} \frac{\partial M}{\partial x_{1}} .
$$

In terms of the original dimensionful parameters, this regime is equivalent to the low-mass-flow (turnover) regime achieved as $\delta \rightarrow 0$. Hence, in the low-mass-flow regime the velocity of a single point sink can be written equivalently in terms of the derivative of total mass $M$ as

$$
\begin{aligned}
\frac{d x_{1}}{d t} & =v \Delta J_{1} \\
& =-v \frac{D}{2} \frac{\partial M}{\partial x_{1}} .
\end{aligned}
$$

In the next subsection, we generalize this relation to $n$ arbitrary sinks.

\section{Dynamics of $\boldsymbol{n}$ sinks}

We consider again the limit $\kappa \ll 1$. Then, the flux differential up to lowest order in $\kappa$ is given by (A14):

$$
\frac{\Delta J_{i}}{c \delta L}=-\frac{n}{L} \frac{\lambda}{1+n \lambda}\left[x_{i}-\frac{L}{n} i+\frac{L}{2}\left(\frac{1}{n}+1\right)\right]+O\left(k^{2}\right),
$$

where we have used the expression for the lowest order term of $\mu^{\prime}$ from (A13):

$$
\mu_{0}^{\prime}=\frac{\lambda c}{1+n \lambda} .
$$

The derivative of $M$ up to second order in $\kappa$ from (A20),

$$
\begin{aligned}
\frac{D}{c \delta L} \frac{\partial M}{\partial x_{i}} & =\left(\frac{\lambda}{1+n \lambda}\right)^{2} \frac{\partial}{\partial x_{i}} \sum_{i, j} G_{2}\left(x_{i} ; x_{j}\right)+O\left(k^{4}\right) \\
& =\frac{2 n}{L}\left(\frac{\lambda}{1+n \lambda}\right)^{2}\left[x_{i}-\frac{L}{n} i+\frac{L}{2}\left(\frac{1}{n}+1\right)\right]+O\left(k^{4}\right),
\end{aligned}
$$

where we have used the second order expansion of $G\left(x_{i} ; x_{j}\right)$ in $\kappa[(\mathrm{A} 20)]$. Taken together, the above equations imply that in the low-mass-flow limit $\delta \rightarrow 0(\kappa \rightarrow 0, \lambda \rightarrow \infty)$

$$
\Delta J_{i}=-\frac{1}{2} n D \frac{\partial M}{\partial x_{i}} .
$$

Therefore, in that limit the dynamics of the point sinks are equivalently specified by

$$
\frac{d x_{1}}{d t}=-v \frac{n}{2} D \frac{\partial M}{\partial x_{i}}
$$

Hence, the dynamics of the system becomes akin to damped particles moving in a potential specified by the total mass. It should be noted that away from this limit, while the dynamics may be different, the two prescriptions share the same stationary points. However, for our parameter choices we found very good agreement between the two systems [Fig. 3(d)].

\section{APPENDIX C: PEAK MOVEMENT AND PATTERN SELECTION IN THE BRUSSELATOR}

We present an analysis of the Brusselator model and show numerically and analytically that most of the features of the exploratory model in (2) still hold. The general spatial version of the Brusselator [51] is described by the following equations:

$$
\begin{aligned}
& \frac{\partial u}{\partial t}=D_{u} \frac{\partial^{2} u}{\partial x^{2}}-\beta u v^{2}+\gamma v, \\
& \frac{\partial v}{\partial t}=D_{v} \frac{\partial^{2} v}{\partial x^{2}}+\beta u v^{2}-\gamma v+c \delta-\delta v .
\end{aligned}
$$

We use reflective boundary conditions at $x= \pm \frac{L}{2}$ and as in (2) write the production term in terms of the degradation rate $\delta$. While the Brusselator also has the form of a mass-conserving Turing system with additional linear terms, here it is the mass of $v$ that is fixed at the steady state,

$$
\frac{1}{L} \int \bar{v} d x=c,
$$


rather than the total mass. As before, we can change the turnover $\delta$ without affecting the steady-state concentration of $v$. We nondimensionalize the system by

$$
x \rightarrow \frac{x}{L}, t \rightarrow \frac{D_{v} t}{L^{2}}, u \rightarrow \frac{u}{c}, v \rightarrow \frac{v}{c}
$$

to obtain

$$
\begin{aligned}
& \frac{\partial u}{\partial t}=d \frac{\partial^{2} u}{\partial x^{2}}+\Gamma\left(-a u v^{2}+v\right), \\
& \frac{\partial v}{\partial t}=\frac{\partial^{2} v}{\partial x^{2}}+\Gamma\left(a u v^{2}-v+b(1-v)\right),
\end{aligned}
$$

where

$$
\Gamma=\frac{\gamma L^{2}}{D_{v}}, d=\frac{D_{u}}{D_{v}}, a=\frac{\beta c^{2}}{\gamma}, b=\frac{\delta}{\gamma} .
$$

There is a single fixed point

$$
u_{0}=\frac{1}{a}, v_{0}=1
$$

The Jacobian is given by

$$
J_{\left(u_{0}, v_{0}\right)}=\Gamma\left[\begin{array}{cc}
-a v_{0}^{2} & -2 a u_{0} v_{0}+1 \\
a v_{0}^{2} & 2 a u_{0} v_{0}-1-b
\end{array}\right]=\Gamma\left[\begin{array}{cc}
-a & -1 \\
a & 1-b
\end{array}\right] .
$$

The Jacobian (and hence the dispersion relation) becomes independent of $b$ for $b \ll 1$, that is when the flow of mass through the system is low, close to the mass-conserved limit $b=0$. Hence, we can change $b$ without significantly affecting the linear behavior of the model. The trace and determinant of the Jacobian are easily found to be

$$
\begin{aligned}
\operatorname{Tr} J & =\Gamma(1-a-b), \\
\operatorname{Det} J & =\Gamma^{2} a b .
\end{aligned}
$$

For the homogeneous fixed point to be stable in the absence of diffusion we must have $\operatorname{Tr} J<0$ and Det $J>0$. Hence, we require $a+b>1$. The Turing condition is given by

$$
d(1-b)-a-2 \sqrt{d a b}>0 .
$$

We numerically solve this system, using reflective boundary condition, by perturbing the homogeneous state as described in numerical methods section. Like in the model of the main text, and every Turing model we are aware of, the peaks of a pattern are periodic and regularly positioned. Furthermore, consistent with our results, a single peak moves exponentially to mid-domain (Fig. S6A [13]). The rate of movement was found to be proportional to $b$ or, equivalently, $\delta$, the turnover rate and at the mass-conserved limit $b=0$ no peak movement is observed.

\section{Spike limit of the Brusselator model}

In this section, we develop the spike limit of the Brusselator model $[35,55]$. Let us consider the dimensional form in $(\mathrm{C} 1)$. As in the case of our toy model we consider the limit $D_{v} \ll$ $D u$, where solutions of $v$ consist of narrow large-amplitude spikes of width $\epsilon=\mathcal{O}\left(\sqrt{D_{v} / \gamma}\right)$. Away from the spikes $v$ is a spatial constant $v_{\text {out }}$. Since $\int_{-L / 2}^{L / 2} v d x=c$, inside the spikes we have $v \gg c$ within a spike and $v=v_{\text {out }} \ll c$ outside. We search for steady-state solutions consisting of $n$ spikes at positions $x_{1}, x_{2}, \ldots, x_{n}$ and assume that $u$ changes slowly within each spike and can be approximated by a constant $u_{i}$. We introduce an inner coordinate $y=\epsilon^{-1}\left(x-x_{i}\right)$, within each spike. The equation for the inner variable $v_{i}(y)$ becomes

$$
\begin{array}{r}
\frac{D_{v}}{\epsilon^{2}} \frac{d^{2} v_{i}}{d y_{i}^{2}}+\beta u_{i} v_{i}^{2}-(\gamma+\delta) v_{i}=0, \\
v_{i} \rightarrow 0 \quad \text { as } \quad y_{i} \rightarrow \pm \infty,
\end{array}
$$

where we have neglected the constant term since $v_{i} \gg c$. The solution to this equation is

$$
v_{i}=\frac{3}{2} \frac{\gamma+\delta}{\beta u_{i}} \operatorname{sech}^{2}\left(\sqrt{\frac{\gamma+\delta}{D_{v}}} \frac{\epsilon y_{i}}{2}\right) .
$$

In the outer region the equation each spike is approximated by a Dirac delta function

$$
v=v_{\text {out }}+\sum_{i} w_{i, 1} \delta\left(x-x_{i}\right)
$$

where $w_{i, 1}$ is the weight

$$
w_{i, 1}=\epsilon \int_{-\infty}^{\infty} v_{i}\left(y_{i}\right) d y_{i}=6 \frac{\sqrt{D_{v}(\gamma+\delta)}}{\beta u_{i}} .
$$

The $\epsilon$ prefactor is the spike width. To write the outer equation for $u$, we also need the weight of the $u v^{2}$ term

$$
w_{i, 2}=\epsilon u_{i} \int_{-\infty}^{\infty} v_{i}^{2}\left(y_{i}\right) d y_{i}=6 \frac{\sqrt{D_{v}}(\gamma+\delta)^{3 / 2}}{\beta^{2} u_{i}} .
$$

Since $\mathcal{O}\left(w_{i, 1}\right)=1$ (spikes have finite weight), we find $\mathcal{O}\left(u_{i n}\right)=\epsilon$ and $\mathcal{O}\left(v_{i n}\right)=\epsilon^{-1}$. Away from the spikes $v$ is a constant $v_{\text {out }}$. Therefore, from (C1b) we have

$$
\beta u v_{\text {out }}^{2}-\gamma v_{\text {out }}+c \delta-\delta v_{\text {out }}=0
$$

in the outer region. Balancing this equation while requiring $u$ and $v_{\text {out }}$ not to diverge requires that $\mathcal{O}(u)<\mathcal{O}\left(v_{\text {out }}\right)$. Therefore, we can neglect the cubic term in the above equation to obtain

$$
v_{\text {out }}=\frac{c \delta}{\gamma+\delta} .
$$

We obtain the outer equation for $u$ by replacing $v$ with weighted delta functions

$$
\begin{gathered}
D_{u} \frac{d^{2} u}{d x^{2}}-\sum_{i=1}^{n}\left[\beta w_{i, 2}-\gamma+w_{i, 1}\right] \quad \delta\left(x-x_{i}\right)+c \delta-\delta v_{\text {out }} \\
-\frac{L}{2}<x<\frac{L}{2}, \quad u_{x}\left( \pm \frac{L}{2}\right)=0,
\end{gathered}
$$

where we used (C5) to simplify contributions from the outer region. Inserting the expressions for each term we arrive at

$$
\begin{array}{r}
D_{u} \frac{d^{2} u}{d x^{2}}-\frac{c \gamma \delta}{\gamma+\delta}-\sum_{i=1}^{n} \frac{\rho}{u_{i}} L \delta\left(x-x_{i}\right)=0, \\
-\frac{L}{2}<x<\frac{L}{2}, \quad u_{x}\left( \pm \frac{L}{2}\right)=0,
\end{array}
$$

where $\rho=6 \frac{\sqrt{D_{v}}}{L} \frac{\delta \sqrt{\gamma+\delta}}{\beta}$. Note the above expression for $v_{\text {out }}$ is consistent with the condition for the integral of $v, L v_{\text {out }}+$ $\sum_{i} w_{i, 1}=c L$, and the integral of the outer equation. 
The solution to the outer equation is

$$
u(x)=\bar{u}-\sum_{j=1}^{n} \hat{\rho}_{i}^{\prime} \hat{G}\left(x ; x_{j}\right)
$$

where $\bar{u}$ is a constant and the Green's function $\hat{G}\left(x ; x_{j}\right)$ is the solution to

$$
\begin{aligned}
-L^{2} \hat{G}_{x x}\left(x ; x_{j}\right)+1 & =L \delta\left(x-x_{j}\right), \quad-\frac{L}{2}<x<\frac{L}{2}, \\
\hat{G}_{x}\left( \pm \frac{L}{2}\right) & =0, \quad \frac{1}{L} \int_{-\frac{L}{2}}^{\frac{L}{2}} \hat{G}\left(x ; x_{j}\right) d x=0
\end{aligned}
$$

given by

$$
\hat{G}\left(x ; x_{j}\right)=\frac{1}{2 L^{2}}\left(x^{2}+x_{j}^{2}\right)-\frac{1}{2 L}\left|x-x_{j}\right|+\frac{1}{12} .
$$

We use hats to distinguish these quantities from those of the model in the main text. The coefficients $\hat{\rho}_{i}^{\prime}=\hat{\rho}_{i}^{\prime}(\mathbf{x})$ and the constant $\bar{u}$ are determined by the nonlinear algebraic system

$$
\hat{\rho}_{i}^{\prime}=\hat{\sigma} \frac{c^{2}}{u\left(x_{i}\right)}, \quad i=1, \ldots, n
$$

where $\hat{\sigma}=\frac{\rho L^{2}}{c^{2} D_{u}}$ is a dimensionless parameter and the consistency condition [obtained by integrating (C7)]

$$
\sum_{i} \hat{\rho}_{i}^{\prime}=c \eta
$$

where $\eta=\frac{\delta}{\gamma+\delta} \frac{\gamma L^{2}}{D_{u}}$ is a second dimensionless parameter.

We next consider the spike limit $\hat{\sigma} \rightarrow 0\left(D_{v} \rightarrow 0\right)$. From (C11) we have

$$
\hat{\rho}_{i}^{\prime}\left(\bar{u}-\sum_{j=1}^{n} \hat{\rho}_{i}^{\prime} \hat{G}\left(x_{i} ; x_{j}\right)\right)=0 \quad \forall i .
$$

As in the model of the main text, if any $\hat{\rho}_{i}^{\prime}=0$, then the result is a solution to the system with one fewer spike. Hence, the only physical $n$-spike solution is

$$
\hat{\boldsymbol{\rho}}^{\prime}=\bar{u} \hat{\mathbf{G}}^{-1} \hat{\mathbf{e}},
$$

where $\hat{\mathbf{G}}_{i j}=\hat{G}\left(x_{i} ; x_{j}\right)$ and $\hat{\mathbf{e}}$ is the column vector with all unit entries. The constant $\bar{u}$ is determined from (C12).

\section{Comparison to point sinks}

Just as for the model of the main text, the solution obtained above is identical to that of a model of perfect point sinks. Consider the following system of (imperfect) point sinks:

$$
D \frac{d^{2} A(x)}{d x^{2}}+\hat{c}-\sum_{i=1}^{n} \mu L \delta\left(x-x_{i}\right) A\left(x_{i}\right)=0
$$

with reflective boundary conditions at $x= \pm \frac{L}{2}$. This is similar to that of (3) but without the background decay ( $\delta u$ term). Indeed, it can be obtained from that equation by replacing $c$ by $\hat{c} / \delta$ and taking the limit $\delta \rightarrow 0$. The solution to this equation is

$$
A(x)=\bar{A}-\sum_{i=1}^{n} \hat{\mu}_{i}^{\prime} \hat{G}\left(x ; x_{i}\right)
$$

where $\bar{A}$ is a constant and the Green's function $\hat{G}\left(x ; x_{i}\right)$ is the same as that of the Brusselator [(C10)]. The coefficients $\hat{\mu}_{i}^{\prime}=$ $\hat{\mu}_{i}^{\prime}(\mathbf{x})$ are determined by the linear algebraic conditions

$$
\hat{\mu}_{i}^{\prime}=\hat{\lambda} A\left(x_{i}\right), \quad i=1, \ldots, n
$$

where we have introduced the dimensionless parameter $\hat{\lambda}=$ $\frac{\mu L^{2}}{D}$. In the perfect sink limit $\hat{\lambda} \rightarrow \infty$ the condition for the $\hat{\mu}_{i}^{\prime}$ 's reduces to

$$
\hat{\boldsymbol{\mu}}^{\prime}=\bar{A} \hat{\mathbf{G}}^{-1} \hat{\mathbf{e}}
$$

which is precisely what we obtained for the Brusselator model in the spike limit. Thus, steady-state spike solutions of the Brusselator are identical to solutions of a model of perfect point sinks.

In Appendix B, for the point-sink model with background decay, we showed a connection [(B6)] between the flux differentials into each sink and the derivative of the total mass $M=\frac{1}{L} \int A(x) d x$. It is easily seen that the same relation holds for the model without background decay. Replacing $c$ by $\hat{c} / \delta$ and taking the limit $\delta \rightarrow 0$, the flux differential across each sink is found from (B4) to be

$$
\frac{\Delta J_{i}}{\hat{c} L}=-\frac{1}{L}\left[x_{i}-\frac{L}{n} i+\frac{L}{2}\left(\frac{1}{n}+1\right)\right] .
$$

Similarly, the derivative of $M$ from (B5) is

$$
\frac{D}{\hat{c} L} \frac{\partial M}{\partial x_{i}}=\frac{2}{n L}\left[x_{i}-\frac{L}{n} i+\frac{L}{2}\left(\frac{1}{n}+1\right)\right] .
$$

Thus,

$$
\Delta J_{i}=-\frac{1}{2} n D \frac{\partial M}{\partial x_{i}} .
$$

Therefore, the dynamics of sinks moving with velocities proportional to the flux differential across them is equivalent to a system of $n$ overdamped particles with the mass $M$ acting as a potential.

\section{Mass minimization predicts the pattern obtained after coarsening}

In Fig. 6, we examine coarsening in the Brusselator model. We use $\Gamma, d, a$, and $b$ as dimensionless parameters and use periodic boundary conditions to avoid patterns with peaks on the boundary (which are not compatible with the spike approximation). In Figs. 6(a) and 6(b), we compare the number of peaks in the dominant mode as predicted by the linear dispersion relation and the number of peaks in the most frequent pattern as obtained from the numerical simulations. We observe a similar coarsening behavior as $b$ (or equivalently the turnover rate $\delta$ ) is decreased, similar to the model of the main text.

To apply our analytical approximation, we consider the situation where spikes are symmetric $\left(\hat{\rho}_{i}^{\prime}=\hat{\rho}_{c}^{\prime}\right)$ and regularly positioned,

$$
\bar{x}_{i}=\frac{L}{n} i-\frac{L}{2}\left(\frac{1}{n}+1\right)
$$


as they are observed to be at steady state. From (C12), we then have

$$
\hat{\rho}_{c}^{\prime}=\frac{c}{n} \eta
$$

We determine the constant $\bar{u}$ by evaluating the solution (C8) at each spike position $\bar{u}_{i}$ :

$$
u\left(\bar{x}_{i}\right)=\frac{n \hat{\sigma}}{\eta} c=\bar{u}-\frac{c \eta}{n} \sum_{j=1}^{n} \hat{G}\left(\bar{x}_{i} ; \bar{x}_{j}\right) .
$$

The sum in the above equation is independent of $i$ since

$$
\sum_{j=1}^{n} \hat{G}\left(\bar{x}_{i} ; \bar{x}_{j}\right)=\frac{1}{12 n} .
$$

Thus, we find that the total mass $M$ of the fast species is

$$
\begin{aligned}
M / c & =\frac{1}{c L} \int_{-\frac{L}{2}}^{\frac{L}{2}} u(x) d x=\frac{1}{c L} \int_{-\frac{L}{2}}^{\frac{L}{2}} \bar{u} d x \\
& =\frac{n \hat{\sigma}}{\eta}+\frac{\eta}{12 n^{2}}
\end{aligned}
$$

where we have used the Green's function property $\int_{-\frac{L}{2}}^{\frac{L}{2}} \hat{G}\left(x ; x_{j}\right)=0$. In terms of the dimensionless parameter set of the first section, the expression for the mass is
$M=\frac{6 n(b+1)^{3 / 2}}{a \sqrt{\Gamma}}+\frac{\Gamma b}{12 d(b+1) n^{2}}$. Note that just as for the model in the main text, the mass $M$ is minimal for a critical number of peaks $n=n_{c}$. In Fig. 6(c), we compare the distribution of peaks of the final pattern obtained after coarsening with $n_{c}$ and the linear prediction. We find that, like in the model of the main text, $n_{c}$ is an excellent predictor of number of peaks in the steady-state pattern.

\section{Absence of a diffusive length scale}

Note that the lack of the background decay term in Eqs. (C7) and (C14) means that unlike for Eqs. (16) and (3), there is no concept of a diffusive length scale in the Brusselator or, rather, the diffusive length scale is infinite as decay occurs only through the point sinks.

In the model of the main text, the parameter $\delta$ (or the dimensionless parameter $\kappa$ ) controlled both the flow of mass through the systems (the limit to a mass-conserved model) and the diffusive length scale. While coarsening increases as the system approaches the mass-conserved limit, it only occurs when the diffusion length scale is longer ( $\kappa$ smaller) than some critical threshold, as this is the point at which peaks begin to compete with each other for the substrate, i.e., $u$.

In the Brusselator, since the diffusive length scale is infinite, peaks always compete and, as a result, coarsening is observed at all values of $\delta$ [compare Fig. 6(c) with 5(e)].
[1] P. Ball, The Self-made Tapestry: Pattern Formation in Nature (Oxford University Press, Oxford, 1999), p. 287.

[2] R. Kapral and K. Showalter, Chemical Waves and Patterns (Springer, Netherlands, 1995).

[3] Y. Kuramoto, Chemical Oscillations, Waves, and Turbulence, Dover Books on Chemistry (Dover, New York, 2003), p. 164.

[4] S. Kondo and T. Miura, Reaction-diffusion model as a framework for understanding biological pattern formation, Science 329, 1616 (2010).

[5] A. J. Koch and H. Meinhardt, Biological pattern formation: from basic mechanisms to complex structures, Rev. Mod. Phys. 66, 1481 (1994).

[6] J. Halatek, F. Brauns, and E. Frey, Self-organization principles of intracellular pattern formation, Philos. Trans. R. Soc., B 373, 20170107 (2018).

[7] E. Meron, Nonlinear Physics of Ecosystems (CRC Press, Boca Raton, FL, 2019).

[8] A. M. Turing, The Chemical Basis of Morphogenesis, Philos. Trans. R. Soc., B 237, 37 (1952).

[9] J. D. Murray, in Mathematical Biology II: Spatial Models and Biomedical Applications, edited by J. D. Murray, Interdisciplinary Applied Mathematics Vol. 18 (Springer, New York, 2003), p. 814.

[10] M. Cross and H. Greenside, Pattern Formation and Dynamics in Nonequilibrium Systems (Cambridge University Press, Cambridge, 2009).

[11] P. K. Maini, T. E. Woolley, R. E. Baker, E. A. Gaffney, and S. Seirin Lee, Turing's model for biological pattern for- mation and the robustness problem, Interface Focus 2, 487 (2012).

[12] S. M. Murray and V. Sourjik, Self-organization and positioning of bacterial protein clusters, Nat. Phys. 13, 1006 (2017).

[13] See Supplemental Material at http://link.aps.org/supplemental/ 10.1103/PhysRevE.103.012215 for background information relevant for the main text, and an analysis of minimal mass in the Schnakenberg model.

[14] M. C. Cross and P. C. Hohenberg, Pattern formation outside of equilibrium, Rev. Mod. Phys. 65, 851 (1993).

[15] A. De Wit, Spatial Patterns and Spatiotemporal Dynamics in Chemical Systems (Wiley, New York, 1999), pp. 435-513.

[16] B. Peña and C. Pérez-García, Selection and competition of Turing patterns, Europhys. Lett. 51, 300 (2000).

[17] B. Peña and C. Pérez-García, Stability of Turing patterns in the Brusselator model, Phys. Rev. E 64, 056213 (2001).

[18] M. E. Cates, D. Marenduzzo, I. Pagonabarraga, and J. Tailleur, Arrested phase separation in reproducing bacteria creates a generic route to pattern formation, Proc. Natl. Acad. Sci. U. S. A. 107, 11715 (2010).

[19] P. Arcuri and J. D. Murray, Pattern sensitivity to boundary and initial conditions in reaction-diffusion models., J. Math. Biol. 24, 141 (1986).

[20] F. Bergmann, L. Rapp, and W. Zimmermann, Active phase separation: A universal approach, Phys. Rev. E 98, 020603(R) (2018).

[21] L. Rapp, F. Bergmann, and W. Zimmermann, Pattern orientation in finite domains without boundaries, Europhys. Lett. 113, 28006 (2016). 
[22] M. Ruppert, F. Ziebert, and W. Zimmermann, Nonlinear patterns shaping the domain on which they live, New J. Phys. 22, 052001 (2020).

[23] F. Bergmann and W. Zimmermann, On system-spanning demixing properties of cell polarization, PLoS One 14, e0218328 (2019).

[24] M. C. Wigbers, F. Brauns, T. Hermann, and E. Frey, Pattern localization to a domain edge, Phys. Rev. E 101, 022414 (2020).

[25] V. K. Vanag and I. R. Epstein, Translational and nontranslational motion of perturbed Turing patterns, Phys. Rev. E 67, 066219 (2003).

[26] M. Dolnik, I. Berenstein, A. M. Zhabotinsky, and I. R. Epstein, Spatial Periodic Forcing of Turing Structures, Phys. Rev. Lett. 87, 238301 (2001).

[27] B. Peña, C. Pérez-García, A. Sanz-Anchelergues, D. G. Míguez, and A. P. Muñuzuri, Transverse instabilities in chemical Turing patterns of stripes, Phys. Rev. E 68, 056206 (2003).

[28] D. G. Míguez, E. M. Nicola, A. P. Muñuzuri, J. Casademunt, F. Sagués, and L. Kramer, Traveling-Stripe Forcing Generates Hexagonal Patterns, Phys. Rev. Lett. 93, 048303 (2004).

[29] L. Yang, M. Dolnik, A. M. Zhabotinsky, and I. R. Epstein, Turing patterns beyond hexagons and stripes, Chaos 16, 037114 (2006).

[30] S. Ishihara, M. Otsuji, and A. Mochizuki, Transient and steady state of mass-conserved reaction-diffusion systems, Phys. Rev. E 75, 015203(R) (2007).

[31] S. Jimbo and Y. Morita, Lyapunov function and spectrum comparison for a reaction-diffusion system with mass conservation, J. Diff. Equ. 255, 1657 (2013).

[32] Y. Morita and T. Ogawa, Stability and bifurcation of nonconstant solutions to a reaction-diffusion system with conservation of mass, Nonlinearity 23, 1387 (2010).

[33] Y. Morita, Spectrum comparison for a conserved reactiondiffusion system with a variational property, J. Appl. Analysis Comput. 2, 57 (2012).

[34] F. Brauns, H. Weyer, J. Halatek, J. Yoon, and E. Frey, Wavelength selection by interrupted coarsening in reaction-diffusion systems, Phys. Rev. Lett. (to be published), arXiv:2005.01495.

[35] J. Tzou, Y. Nec, and M. Ward, The stability of localized spikes for the 1-D Brusselator reaction-diffusion model, Eur. J. Appl. Math. 24, 515 (2013).

[36] T. Kolokolnikov, M. J. Ward, and J. Wei, The Existence and Stability of Spike Equilibria in the One-dimensional Gray-Scott model: The low feed-rate regime, Stud. Appl. Math. 115, 21 (2005).

[37] T. Kolokolnikov, M. J. Ward, and J. Wei, Slow translational instabilities of spike patterns in the one-dimensional Gray-Scott model, Interfaces Free Boundaries 8, 185 (2006).

[38] M. Otsuji, S. Ishihara, C. Co, K. Kaibuchi, A. Mochizuki, and S. Kuroda, A Mass Conserved Reaction-Diffusion System Captures Properties of Cell Polarity, PLoS Comput. Biol. 3, e108 (2007).

[39] E. J. Crampin, E. A. Gaffney, and P. K. Maini, Reaction and diffusion on growing domains: scenarios for robust pattern formation., Bull. Math. Biol. 61, 1093 (1999).

[40] T. Sugawara and K. Kaneko, Chemophoresis as a driving force for intracellular organization: Theory and application to plasmid partitioning, Biophysics 7, 77 (2011).
[41] R. Ietswaart, F. Szardenings, K. Gerdes, and M. Howard, Competing ParA structures space bacterial plasmids equally over the nucleoid., PLoS Comput. Biol. 10, e1004009 (2014).

[42] J. Wei and M. Winter, Applied Mathematical Sciences, Applied Mathematical Sciences Vol. 189 (Springer, London, 2014), pp. $1-339$.

[43] K. Kawasaki and T. Ohta, Kink dynamics in one-dimensional nonlinear systems, Phys. A (Amsterdam) 116, 573 (1982).

[44] T. Kawahara and S. Toh, Pulse interactions in an unstable dissipative-dispersive nonlinear system, Phys. Fluids 31, 2103 (1988).

[45] S.-I. Ei and T. Ohta, Equation of motion for interacting pulses, Phys. Rev. E 50, 4672 (1994).

[46] S. I. Ei, The motion of weakly interacting pulses in reactiondiffusion systems, J. Dynam. Diff. Equations 14, 85 (2002).

[47] H. Yamada and K. Nozaki, Interaction of pulses in dissipative systems: FitzHugh-Nagumo equations, Prog. Theor. Phys. 84, 801 (1990).

[48] T. Ohta and T. Yoshimura, Statistical pulse dynamics in a reaction-diffusion system, Phys. D (Amsterdam) 205, 189 (2005).

[49] A. Doelman, W. Eckhaus, and T. J. Kaper, Slowly modulated two-pulse solutions in the Gray-Scott model I: Asymptotic construction and stability, SIAM J. Appl. Math. 61, 1080 (2000).

[50] A. Hofmann, J. Mäkelä, D. J. Sherratt, D. Heermann, and S. M. Murray, Self-organised segregation of bacterial chromosomal origins, eLife 8, e46564 (2019).

[51] I. Prigogine and R. Lefever, Symmetry breaking instabilities in dissipative systems. II, J. Chem. Phys. 48, 1695 (1968).

[52] J. Schnakenberg, Simple chemical reaction systems with limit cycle behaviour, J. Theor. Biol. 81, 389 (1979).

[53] J.-C. Walter, J. Dorignac, V. Lorman, J. Rech, J.-Y. Bouet, M. Nollmann, J. Palmeri, A. Parmeggiani, and F. Geniet, Surfing on Protein Waves: Proteophoresis as a Mechanism for Bacterial Genome Partitioning, Phys. Rev. Lett. 119, 028101 (2017).

[54] D. Iron, J. Wei, and M. Winter, Stability analysis of Turing patterns generated by the Schnakenberg model, J. Math. Biol. 49, 358 (2004).

[55] J. C. Tzou, A. Bayliss, B. J. Matkowsky, and V. A. Volpert, Stationary and slowly moving localised pulses in a singularly perturbed Brusselator model, Eur. J. Appl. Math. 22, 423 (2011).

[56] J. Halatek and E. Frey, Rethinking pattern formation in reaction-diffusion systems, Nat. Phys. 14, 507 (2018).

[57] F. Brauns, J. Halatek, and E. Frey, Phase-Space Geometry of Mass-Conserving Reaction-Diffusion Dynamics, Phys. Rev. X 10, 041036 (2020).

[58] A. Pogan and A. Scheel, Layers in the Presence of Conservation Laws, J. Dynam. Different. Equations 24, 249 (2012).

[59] M. Kuwamura and Y. Morita, Perturbations and dynamics of reaction-diffusion systems with mass conservation, Phys. Rev. E 92, 012908 (2015).

[60] T. Kolokolnikov, M. J. Ward, and J. Wei, The existence and stability of spike equilibria in the one-dimensional Gray-Scott model: The pulse-splitting regime, Phys. D (Amsterdam) 202, 258 (2005).

[61] A. Gierer and H. Meinhardt, A theory of biological pattern formation, Kybernetik 12, 30 (1972). 
[62] D. Iron, M. J. Ward, and J. Wei, The stability of spike solutions to the one-dimensional Gierer-Meinhardt model, Phys. D (Amsterdam) 150, 25 (2001).
[63] Periodic Reaction-Diffusion PDE solver, MATLAB Central File Exchange, https://www.mathworks.com/matlabcentral/ fileexchange/45955-periodic-reaction-diffusion-pde-solver. 